\title{
INFRARED ABSORPTION LINES IN BORON-DOPED SILICON
}

by

KONRAD COLBOW

B.Sc。, McMaster University, Hamilton, Ont., 1959

M.Sc., McMaster University, Hamilton, Ont., 1960

A THESIS SUBMItTED IN PARTIAL FULFILMENT OF

THE REQUIREMENTS FOR THE DEGREE OF́

DOCTOR OF PHILOSOPHY

in the Department

of

PHYSICS

We accept this thesis as conforming to the required standard

THE UNIVERSITY OF BRITISH COLUMBIA

May, 1963 
In presenting this thesis in partial fulfilment of the requirements for an advanced degree at the University of British Columbia, I agree that the Library shall make it freely available for reference and study. I further agree that permission for extensive copying of this thesis for scholarly purposes may be granted by the Head of my Department or by his representatives. It is understood that copying or publication of this thesis for financial gain shall not be allowed without my written permission.

Department of Physics The University of British Columbia, Vancouver 8, Canada. Date May 3, -1963 
The University of British Columbia

- FACULTY OF GRADUATE STUDIES

PROGRAMME OF THE

FINAL ORAL , EXAMINATION

FOR THE DEGREE OF

DOCTOR OF PHILOSOPHY

of

\section{KONRAD COLBOW}

B.Sc., McMaster University, 1959

M.Sc., McMaster University, 1960

WEDNESDAY, MAY 29, 1963, AT 2:000 P.M.

IN ROOM 452, BUCHANAN BUILDING

COMMITTEE IN CHARGE

Chairman: F.H: Soward

R. Barrie

A.M. Crooker

F.W. Dalby
J.C. Giles

K. B. Harvey G.M. Volkoff

External Examiner: H.J. Hrostowski

Department of Physics,

University of Oregon 


\section{INFRARED ABSŌRPTION LINES IN}

\section{BORON-DOPED SILICON}

\section{ABSTRACT}

In boron-doped silicon, optical absorption takes place through the excitation of bound holes from the ground $\$$ tate to excited states. This leads to a line spectrum. Due to a lack of sufficient resolution and a failure to make proper allowance for line distortion by the finite spectrometer slit width, previous authors gave a misleading picture of the low temperature half-width, the temperature: dependence of this halfwidth, and the onset of concentration broadening at low temperatures.

New experimental data are presented and explained by introducing the mechanism of statistical Stark broadening due to ionized impurities, and by modifying Baltensperger's (1953) theory for concentration broadening. At low impurity concentration the width is attributed to phonon broadening (Barrie and Nishikawa 1962) and internal strains (Kohn 1957)。

\section{GRADUATE STUDIES}

Field of Study: Physics

Physics of the solid state

R. Barrie

Dielectrics and Magnetism

M. Bloom

Noise in Physical Systems

R。E. Burgess

Electron Dynamics

R。E。Burgess

Related Studies:

Applied Electromagnetic Theory

G.B。Walker

Digital Computer Programming

J . R.H。Dempster

\section{PUBLICATIONS}

1. Temprature Dependence of Absorption Line Width in Boron-Doped Silicon。 Konrad Colbow, J.W. Bichard, and J.C. Giles。Can。J. Phys. 40, 1436 (1962)。

2. Absorption Line Width in Boron-Doped Silicon. Konrad Colbow.Bul1.Am. Phys,Soc.Series II, I, 485 (1962)。 


\section{ABSTRACT}

In boron-doped silicon, optical absorption takes place through-the excitation of bound holes from the ground state to excited states. This leads to a line spectrum. Due to a lack of sufficient resolution and a failure to make proper allowance for line distortion by the finite spectrometer slit width, previous authors gave a misleading picture of the low temperature halfowidth, the temperature dependence of this halfowidth, and the onset of concentration broadening at low temperatures.

New Ifke experimental data are explained by introducing the mechanism of statistical stark broadening due to ionised impurities, and by modifying Baltensperger's (1953) theory for concentration broadening. At low impurity concentration the width is attributed to phonon broadening (Barrie and Nishikawa 1962) and internal strains (Kohn 1957)。 


\section{ACKNOWLEDGEMENTS}

My sincere thanks are due to Dr. R. Barrie for helpful advice and constructive criticism during the preparation of this thesis.

I also wish to thank Drs. J. W. Bichard, J. C. Giles, and A. M. Crooker for valuable discussions。

The research for this thesis was supported by the Defence Research Board of Canada, Grant No. 9512-26. I also like to express my gratitude to the National Research Council of Canada for the award of a studentship. 
Abstract . . . . . . . . . . . . . . . . .

Table of Contents ....................

List of figures . . . . . . . . . . . . . . . . v v

Acknowledgements ..................... . . vii

Chapter I - Introduction

Chapter II - Experimental

1. Apparatus and Experimental Procedure ....... 4

2. Spectrometer Broadening ........... 7

3. Results and Discussion of Errors ........ 12

Chapter III - Theory and Interpretation of Data

1. Impurity States and the Hydrogenic Approximation .

2. General Interpretation of Line Broadening . . . 20

3. Statistical Theory of Stark Broadening . . . . . 21

4. The Effect of Screening . . . . . . . . . 26

5. Temperature Dependence of the Half-width due to the Linear and the Quadratic Stark Effect . . . .

6. Evaluation of the Half-width due to the Quadratic Stark Effect ............... 29

7. Statistical Broadening due to van der Waals Forces. 31

8. Broadening due to Overlap Forces . . . . . . 32

9. Broadening due to Internal Strains ....... 35

10. Phonon Broadening ............ 36 
Table 1: Standard Voigt profiles ............. 43

Table 2: Integrated absorption, A .......... 44

Table 3: Integrated absorption crossosection, . . . . . 44

Appendix I: Distribution of holes between the ground state, excited states, and the valence band as a function temperature .................

Appendix II: Second-order Stark shift for hydrogenic 1 s and $2 \mathrm{p}$ states. ................ 


\section{LIST OF FIGURES}

To follow

page:

FIG. 1. Absorption constant vs, wave number for boron-doped

silicon at various temperatures and three boron

concentrations

FIG. 2. Water vapor line-width vs, wave number . . . . 8

FIG. 3. Typical Voigt analysis (water vapor line at $298.6 \mathrm{~cm}^{-1}$ )。 10

FIG. 4. Extrapolation of the true half-width to zero

concentration of impurities . . . . . . . .

FIG. 5. True halfowidth vs, temperature for three boron concentrations

FIG. 6. Half-width minus zero-concentration half-width vs. temperature for line $4 \quad$. . . . . . . . .

FIG. 7. Absorption constant vs, wave number for $11 \mathrm{ohm} \mathrm{cm}$ boron-doped silicon at four temperatures . . . . 12

FIG. 8. Energy level diagram for boron-doped silicon . . . 14

FIG. 9. Distribution of holes between the ground state, excited states, and the valence band vs, temperature. 14

FIG. 10. Screened electric field, and Coulomb field with cutoff, as a function of separation . . . . . . .

FIG. 11. Screening parameter vs. temperature for three concentrations of boron .............

FIG. 12. Field distribution function for various values of the screening parameter.............. 
To follow page:

FIG. 13. Half-width of the field distribution as a function of the screening parameter ............

FIG. 14. Half-width in units of the strength parameter vs.

temperature for the linear Stark effect . . . . 28

FIG. 15. Half-width in units of the strength parameter vs.

temperature for the quadratic Stark effect .....

FIG. 16. Broadening of hydrogenic levels vs. distance between impurities in units of the effective Bohr radius . . 
CHAPTER I

INTRODUCTION

The introduction of a group III impurity, like boron, into a perfect silicon. lattice produces a hole (electron deficiency), loosely bound to the impurity ion. In boron-doped silicon, infrared absorption takes place through the excitation of bound holes from the ground state to excited states. This leads to a line spectrum. If the bound hole states were unaffected by, each other and by lattice vibrations, these spectral lines would have no width other than their natural width, which is about $10^{-7}$ electron volts (ev), (e.g Hexzberg 1944). However, the lines that have been observed are considerably broader. This gives rise to two interesting fields of study, namely line broadening due to interaction with the lattice vibrations, and so-called concentration broadening, that is broadening due to the interaction of bound holes with each other. Most of the discussions in this thesis apply in a similar way to group $V$ donor impurities (bound electrons). The reason for choosing a more complicated acceptor impurity like boron is purely historical.

Neglecting instrumental broadening, Burstein et al (1953) found in boron-doped silicon half-widths of about $1 \mathrm{mev}\left(10^{-3} \mathrm{ev}\right)$ at $4.2^{\circ} \mathrm{K}$ and an increase of the half-width (full width at half-maximum) of about 40 percent at $.77^{\circ} \mathrm{K}$. This was later concluded to be in reasonable agreement with the calculations of Lax and Burstein (1955) which gave a halfowidth of $3.6 \mathrm{mev}$ at $4.2{ }^{\circ} \mathrm{K}$ and predicted roughly the observed increase in half-width at $77^{\circ} \mathrm{K}$. Lax and Burstein (1955) suggested that the width of the impurity levels is due to the simultaneous emission or absorption of one or more phonons accompanying the change of state of the'bound hole. Sampson and Margenau (1956) further improved on the agreement between the calculated and the 
observed widths. Using the simple Lorentz broadening approach they calculated a low temperature half-width of $1.6 \mathrm{mev}$.

Kane (1960) pointed out that the observed broadening at $4.2{ }^{\circ} \mathrm{K}$ may be entirely instrumental. Kane suggested that due to the weakness of the electron-phonon interaction in silicon and germanium the dominant optically induced transition is purely electronic without any change of phonon occupation number. He suggested that the width arose from a finite lifetime of the excited state due to the electron-phonon interaction。 Kane estimated a width of about $3 \times 10^{-3}$ mev. In this life-time broadening, the widths of the absorption lines are mostly determined by those of the excited levels. Thus the width of the lines will critically depend on the energy level structure, and one expects different lines to have different widths. This was in disagreement with the experimental results of Burstein et al (1953), and the theory by Lax and Burstein (1955), which predicted that the widths were mostly determined by the ground state and thus should be the same for all lines.

Colbow et al (1962) showed that the observed broadening at $4.2{ }^{\circ} \mathrm{K}$ is not entirely instrumental as suggested by Kane (1960)。However, taking proper care of instrumental broadening they found a half-width of 0.2 mev at 4.2 ${ }^{\circ} \mathrm{K}$ and an increase of the half-width between $4.2{ }^{\circ} \mathrm{K}$ and $90^{\circ} \mathrm{K}$ by a factor of 6, rather than the 40 percent predicted by Lax and. Burstein (1955). Their data also showed that different lines have different half-widths, and that the line shape at $4.2{ }^{\circ} \mathrm{K}$ is predominantly lorentzian.

The theory of phonon broadening has been clarified by Barrie and Nishikawa (1962)。Their results are in full agreement with Kane's (1960). Barrie and Nishikawa obtained explicitly the line shape function of the zerophonon process, which turns out to be approximately lorentzian near the peak. 
They pointed out that the multiphonon processes which Lax and Burstein (1955) considered contribute to the continuous background and become important only at higher temperatures (about $90^{\circ} \mathrm{K}$ )。 However, the theory of Barrie and Nishikawa did not account for the rather steep temperature dependence observed by Colbow et al (1962).

Up to this point it had always been assumed that for the rather low impurity concentrations used by Colbow et al (1962) and Burstein et al (1953), concentration broadening was negligible. This appeared to follow from the experimental data of Newman (1956), which showed that for borondoped silicon concentration broadening starts above $10^{16}$ boron impurities per $\mathrm{cm}^{3}$ 。 In addition Baltensperger's (1953) calculations suggested that concentration broadening in boron-doped silicon should start at approximately $6 \times 10^{16}$ boron impurities per $\mathrm{cm}^{3}$. In contrast to this, the data presented here show that at an impurity concentration of $1.2 \times 10^{15} \mathrm{~cm}^{-3}$ concentration broadening is already important. It is believed that the main shortcoming of Baltensperger's (1953) theory lies in the assumption of a regular lattice of impurities. The implications of an alternative, and more likely assumption of a random distribution of impurities are discussed in this thesis.

A new mechanism is suggested to account for the observed rapid increase of the half-width with temperature, namely statistical stark broadening due to Ionized impurities. 
CHAPTER II

1. Apparatus and Experimental Procedure

Radiation from a Globar source was dispersed in a Model 83 PerkinElmer spectrometer modified to house a Bausck and Lomb grating with 30 grooves per millimeter blazed at $333 \mathrm{~cm}^{-1}$ in the first order. The theoretical resolving power was $\lambda / \Delta \lambda=315$, for a slit width of $0.8 \mathrm{~mm}$. The intensity of shorter wavelength radiation was reduced by sooting the mirror in the entrance optics and by using two reflections from sodium fluoride residual ray plates. The remaining short wavelength radiation was measured and corrected for by passing the radiation through a Rocksalt window, which will transmit only radiation shorter than about $500 \mathrm{~cm}^{-1}$. The effect of the short wavelength photons on the occupation number of the impurity states is negligible. The short wavelength radiation merely contributes to the continuous background, and was within experimental error the same through a pure and the doped sample. Any possible heating of the specimen due to this radiation is negligible as well. Near the entrance slit the radiation is chopped by a semi-circular disc at a frequency of 13 cycles $\mathrm{sec}^{-1}$. After detection by a thermocouple with a cesium lodide window, the amplified signal. was displayed on a Brown strip chart recorder. In each burst of light the number of photons is very small compared to the total number of boron impurities in the sample. During the time interval when the radiation is stopped by the chopper blade, the sample is believed to return to equilibrium. Thus no saturation effects are expected to occur.

The spectrometer was calibrated by means of the atmospheric water vapor absorption spectrum, using the data of Plyler and Acquista (1956), and Randall et al (1937)。 In the course of a measurement the spectrometer was flushed continuousiy, with dry nitrogen gas to reduce the absorption due to

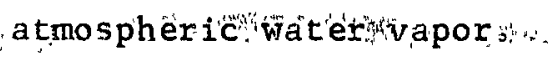


The experiment consists of measuring the transmission ratios of boron-doped silicon and intrinsic silicon as a function of the wave number of the radiation for various temperatures and resistivities of boron-doped silicon. The measurements were made at four temperatures corresponding to the boiling points of helium $\left(4.2^{\circ} \mathrm{K}\right)$, nitrogen $\left(77.4^{\circ} \mathrm{K}\right)$, oxygen $\left(90.1^{\circ} \mathrm{K}\right)$, and pumped nitrogen $\left(60+3^{\circ} \mathrm{K}\right)$.

The refrigerants were contained in a metal dewar vessel, the base of which was located near a focus of the radiation leaving the monochromator. The radiation ports were covered with cesium iodided windows. Parallelsided specimens were attached to a flat copper block at the base of the coolant container, with facilities for rotating alternatively the doped or an intrinsic sample into the path of the radiation. Thermal contact between the copper block and the samples was achieved by means of vacuum grease containing a suspension of silver powder. The samples were held in place by a flat copper strip (with a hole in its center for the passage of the radiation). Carewas paid to avoid straining the samples.

The samples were cut from ingots grown by Merck and Company by the floating-zone technique. They had thicknesses between $0.0361 \mathrm{~cm}$ (most highly doped material) and $0.5085 \mathrm{~cm}$ (1ightly doped material), the latter being the largest thickness that could be readily accommodated in the metal dewar available. These thicknesses were chosen to give a compromise between the signal strength (high transmission) and the observable absorption (low transmission). The specimen surfaces were ground with progressively finer grades of carborundum and finally polished with levigated alumina. Before mounting in the dewar vessel, the surfaces, were degreased in an ultrasonic cleaning bath. Impurity concentrations were determined from the room-temperature resistivity of each sample. The resistivities were 1.3 ohm cm, $11 \mathrm{ohm} \mathrm{cm}, 130 \mathrm{ohm} \mathrm{cm}$, and $3600 \mathrm{ohm} \mathrm{cm}$ (intrinsic). All samples were 
essentially uncompensated. Using the data of Irvin (1962) and Morin and Maita (1954), the corresponding concentrations of boron are respectively $1.2 \times 10^{16} \mathrm{~cm}^{-3}, 1.2 \times 10^{15} \mathrm{~cm}^{-3}, 1.0 \times 10^{14} \mathrm{~cm}^{-3}$, and less than $5 \times 10^{12}$ $\mathrm{cm}^{-3}$ (intrinsic)。

With one exception, the specimens used in the present investigation were thick enough that interference fringes could not be observed with the resolution available. For the one specimen $(0.0361 \mathrm{~cm}$ thick, $1.3 \mathrm{ohm} \mathrm{cm}$ ) for which interference fringes did appear, they were narrow and shallow enough to be readily averaged out in reading the data of the recorder chart. Under these conditions, the monochromatic absorption coefficient $\alpha$ is obtained from the transmission at a given wave number by (Moss 1959)

$$
T=\frac{(1-R)^{2} \exp \cdot(-\alpha d)}{1-R^{2} \exp (-2 \alpha d)}
$$

The transmission of the intrinsic materlal is then given by

$$
\mathrm{T}_{\mathrm{O}}=(1-\mathrm{R})^{2} /\left(1-\mathrm{R}^{2}\right) \text {. }
$$

Hence the transmission ratio of doped and intrinsic silicon becomes

$$
T / T_{0}=\frac{\left(1-R^{2}\right) \exp (-\alpha d)}{1-R^{2} \exp (-2 \alpha d)} \text {. }
$$

This is the ratio that was actually measured in a typical experiment. Here $d$ is the specimen thickness. The surface reflectivity $R$ was assumed to be the same for the intrinsic and the boron-doped silicon。 Using Eq. (1 - 2) a value of $0.31+0.03$ was determined for $R$, which is the same as that used by Bichard and Giles (1962)。 Within experimental error the reflectivity remained constant over the frequency region under study $\left(360 \mathrm{~cm}^{-1}\right.$ to 240 $\left.\mathrm{cm}^{-1}\right)$, and the temperature range $\left(4.2^{\circ} \mathrm{K}\right.$ to $\left.90^{\circ} \mathrm{K}\right)$ investigated.With this value for the reflectivity Eq.(1-3) leads to the graphs of absorption 
FIGURE 1.

Absorption constant vs. wave number for boronodoped

silicon at various temperatures ( $T$ ) and three boron concentrations $\left(\mathrm{N}_{\mathrm{A}}\right)$.

To follow page 6 . 


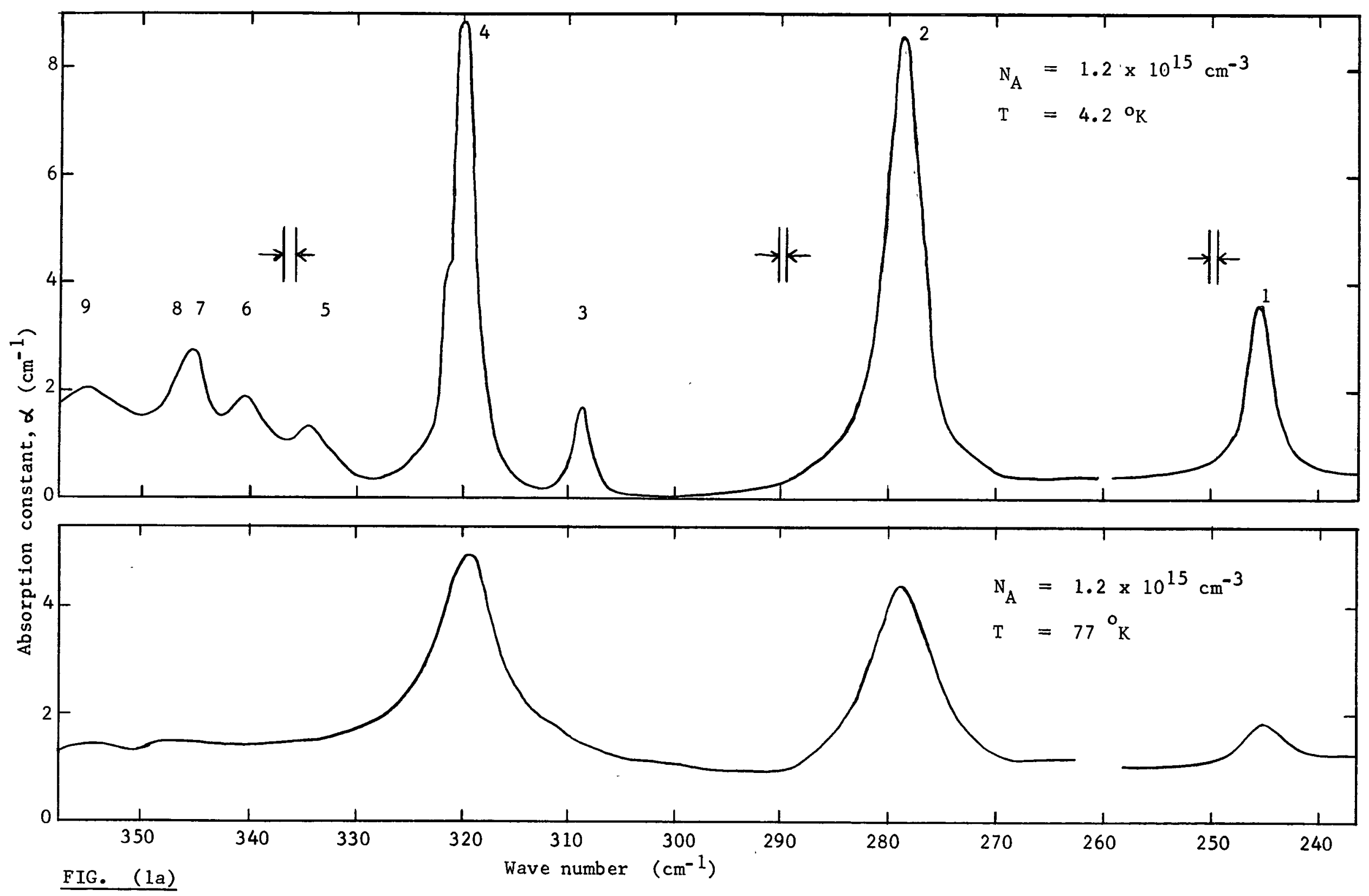




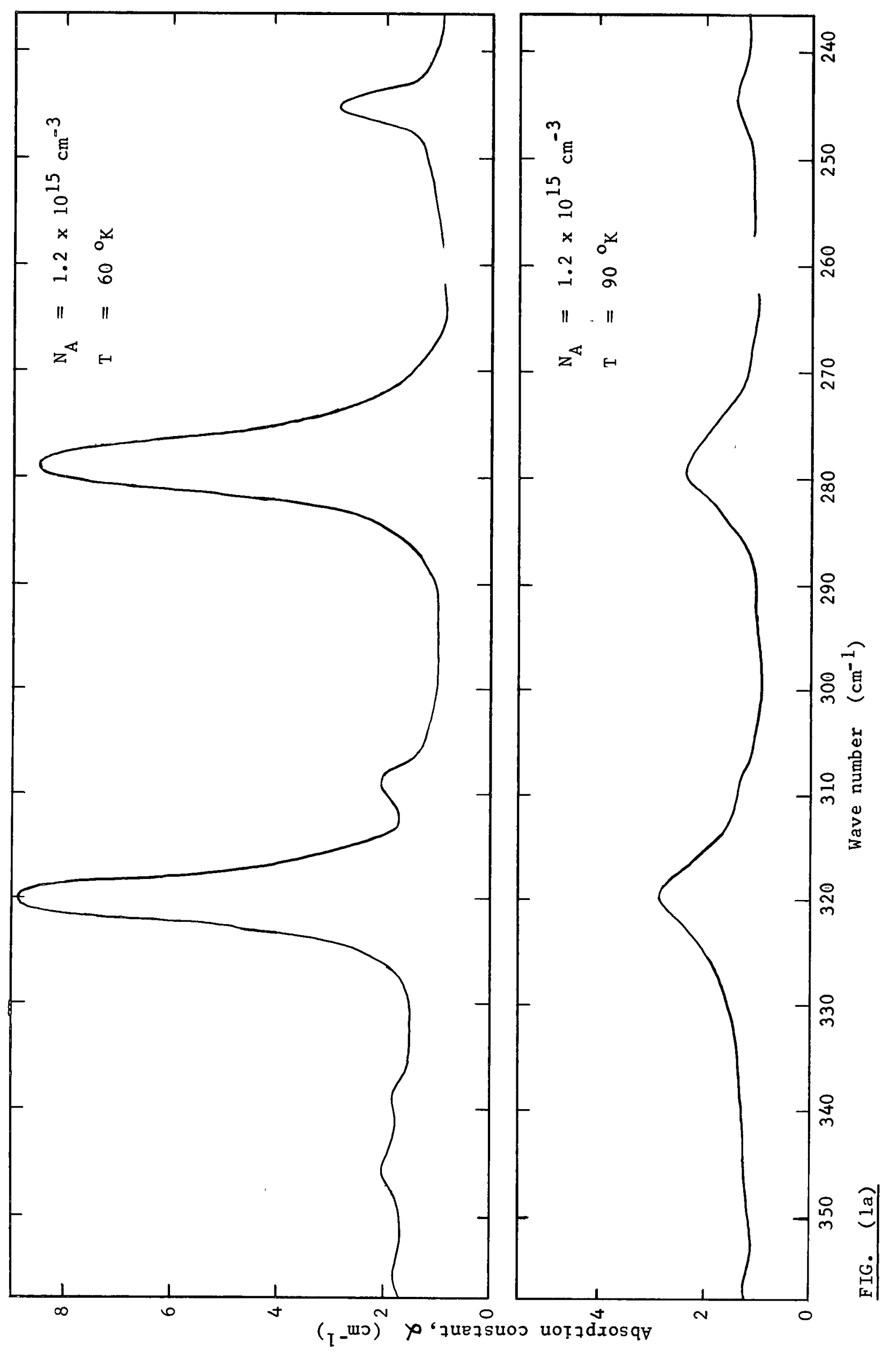




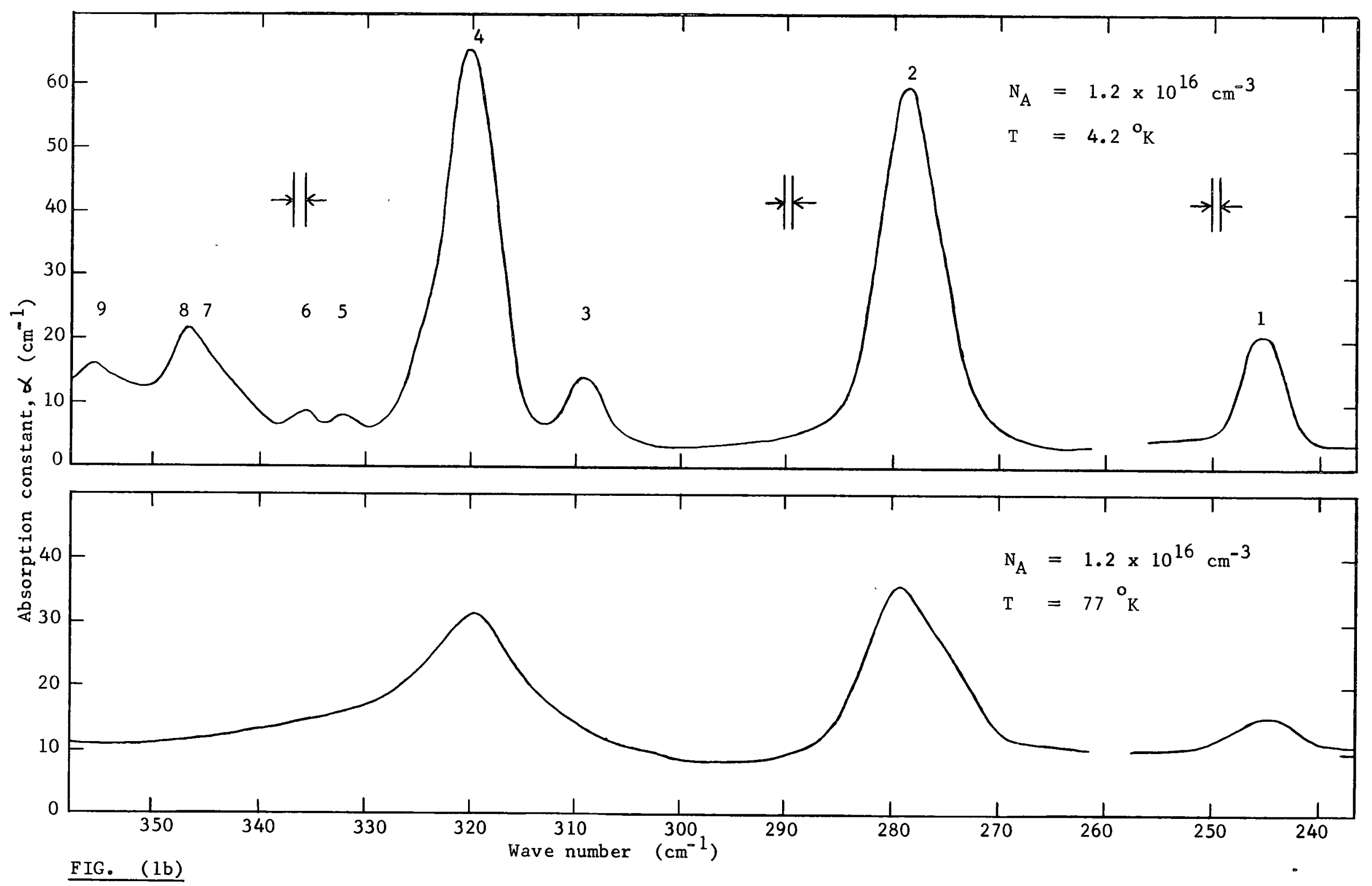




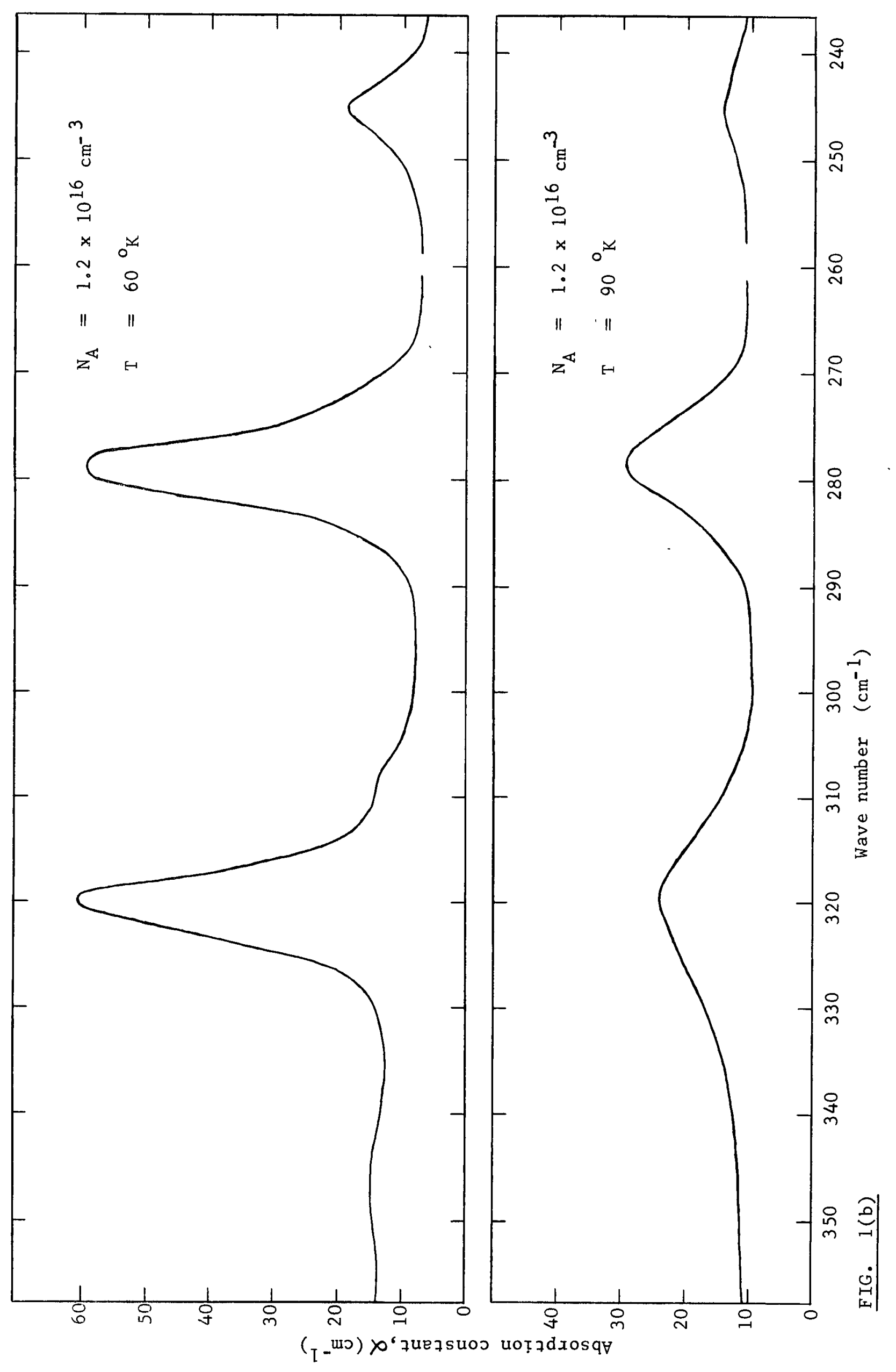



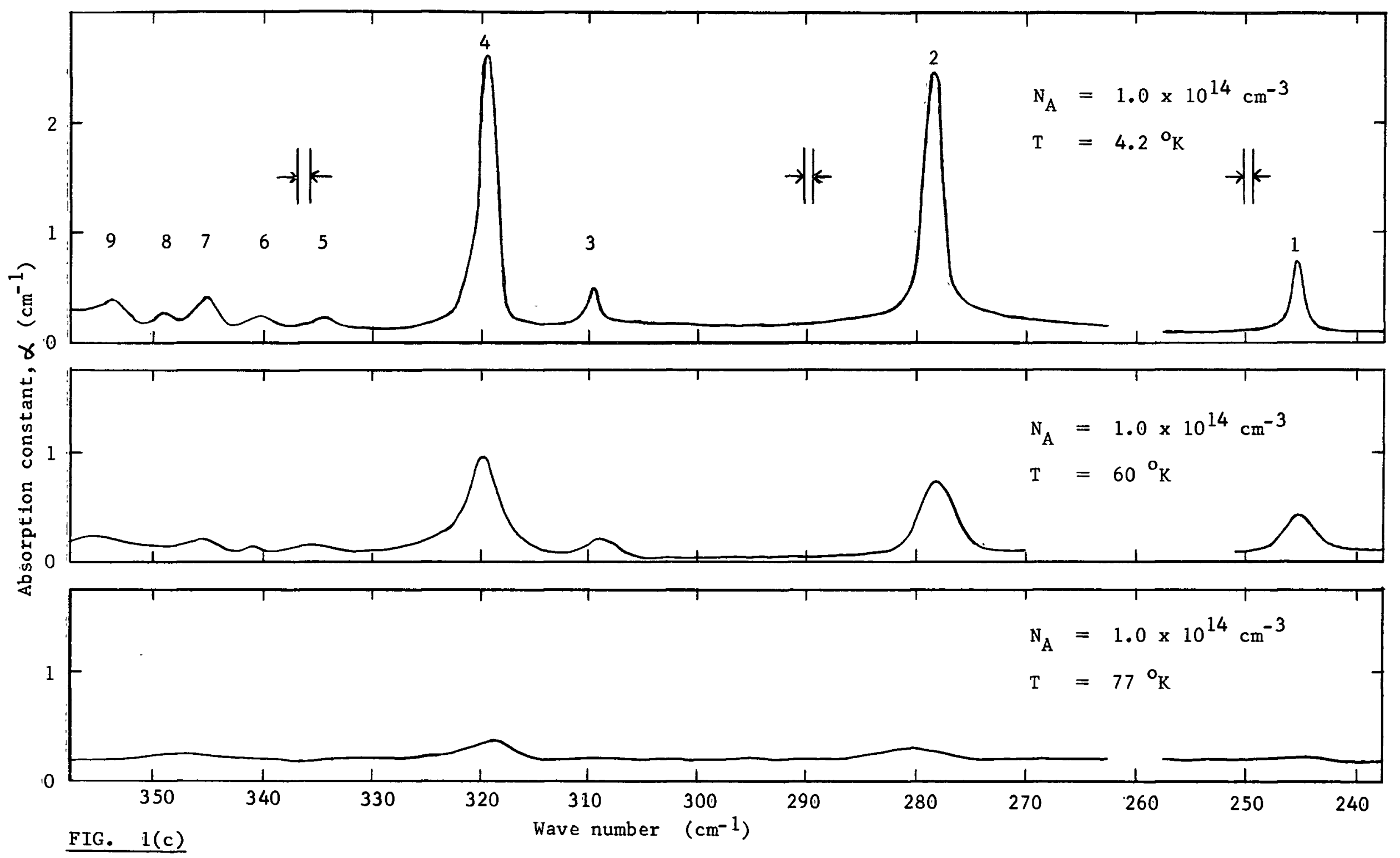
constant as a function of wave number shown in. Figure 1. Instead of measuring the transmission for a doped sample relative to that for an intrinsic sample and using Eq. (1-3), one could have determined the transmission for a doped sample relative to air, using Eq.(1-1)。 However, there are several advantages to the first method. The exact value for the reflectivity is considerably less important for calculating the absorption coefficient if Eq.(1-3), rather than $\mathrm{Eq},(1-2)$, is used. In addition, scattered short wavelength radiation can be expected to be more nearly the same and thus cancel in the ratio, when the doped sample is compared with an intrinsic one, rather than with no sample in the light path. The same applies for the cancel lation of atmospheric water vapor absorption.

In the preceeding outline for the calculation of the absorption coefficient it was implicitly assumed that a parallel beam of light is at normal incidence on a parallel-sided specimen. Any angles between the specimen surfaces were less than $0.01^{\circ}$. Actually, the light beam was not parallel. However, since the maximum angle of incidence in the converging beam was only $8^{\circ}$, no correction for convergence needed to be applied.

\section{Spectrometer Broadening}

Using the curves of Figure 1 and "peeling off" the contributions of neighboring lines, one can determine an. "observed line shape" and an "observed halfowidth". The peeling off is achieved by assuming a symmetric line profile. The resulting absorption lines (dotted lines) may be seen in Figure 6, which gives a magnified picture of the lines 3 and 4 of Figure 1(a). This "peeling off" becomes difficult at higher temperatures. However, if one assumes that the areas under the weaker lines in Figure 6 stay nearly constant and that their heights decrease with temperature in the same way as the strong absorption line, one can decompose the experimental curves into 
the dotted lines, as shown in Figure 6. Errors in these assumptions give rise to considerably smaller errors' in the observed half-width of the strong line。 These observed absorption lines are not only broadened by processes in the specimen but also by the finite resolution of the spectrometer. It is the purpose of the present section to outline the method used for obtaining from the observed line the "true line shape" and the "true half-width", that is the line profile obtained by using a spectrometer with infinite resolution. The intensity distribution in a spectral line broadened by two independent effects is expressed by a convolution integral of the form (Unsöld 1955)

$$
f(x)=\int_{-\infty}^{+\infty} f^{\prime}(x-y) f^{\prime \prime}(y) d y .
$$

Here $f^{\prime}(x)$ or $f^{\prime \prime}(x)$ is the profile the line would assume if only one broadening effect were present; $x$ is the distance from the centre of the line in terms of either wavelength or frequency units. If $f(x)$ is the observed line shape, Eq.(2-1), determines the true line, profile $f^{\prime \prime}-(y)$, provided we know the slit function $f^{\prime}(y)$. This slit function is the profile a mohochromatic signal assumes after passing through the spectrometer. It may be obtained experimentally from the observed profiles of single atmospheric water vapor absorption lines, which have a true width considerably. smaller than the observed width and thus approximate well enough to monochromatic lines. The small true width of single water vapor lines was inferred from a plot, of their observed half-width as a function of spectrometer slit width. The observed half-widths of suitable water vapor absorption lines have been plotted in Figure 2. The corresponding spectrometer slit width is $0.8 \mathrm{~mm}$ in the region from $370 \mathrm{~cm}^{-1}$ to $260 \mathrm{~cm}^{-1}$, and $1.2 \mathrm{~mm}$ in the region from $260 \mathrm{~cm}^{-1}$ to $240 \mathrm{~cm}^{-1}$. The same slit widths were used to obtain the spectra in Figure 1 。 
FIGURE 2.

Water vapor line-width vs. wave number.

To follow page 8 . 


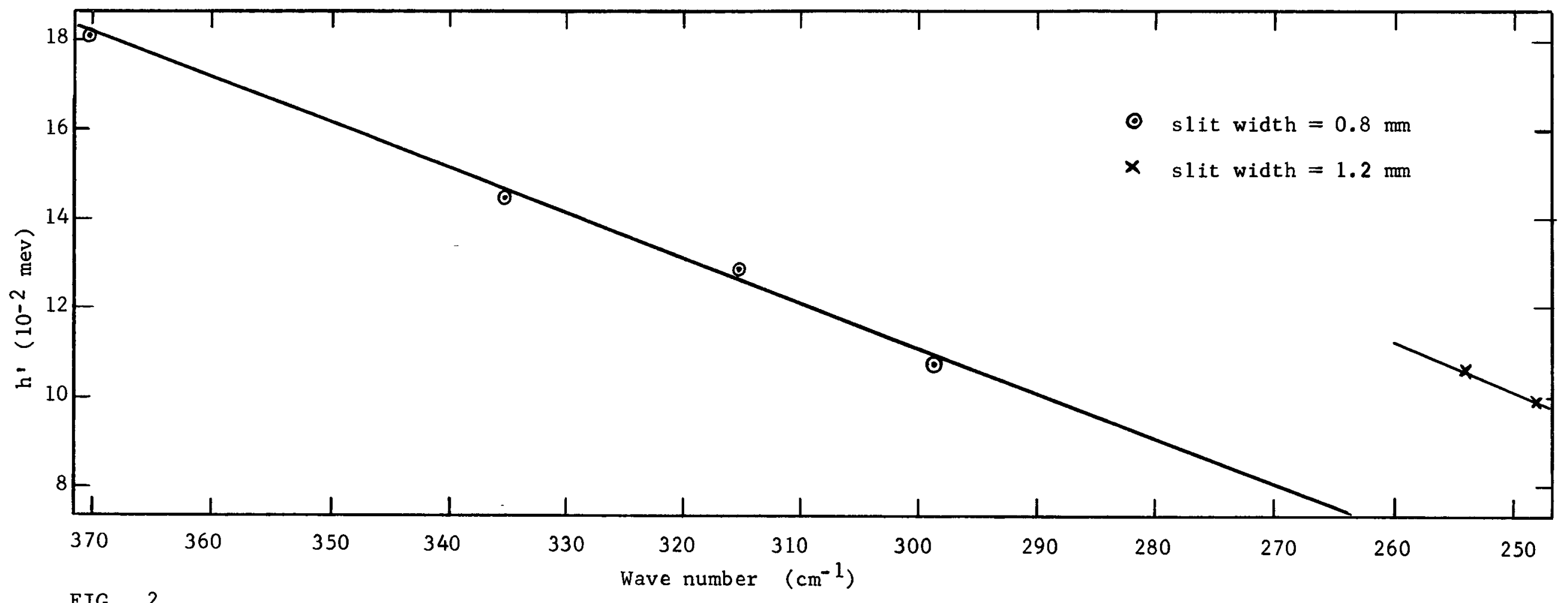

FIG. 2 
Numerous methods for solving the integral equation (2-1) have been applied (Uns81d 1955, van de Hulst 1946). However, all the general methods are very laborious. The problem becomes quite simple if the slit function and the observed line profile may be fitted either both by lorentzian or both by gaussian curves. In this case the true line profile comes out to be respectively lorentzian or gaussian as well, and one has the simple relation between the half - widths

$$
\begin{aligned}
& \text { lorentzian: } h^{\prime \prime}=h-h^{\prime} \\
& \text { gaussian: } h^{\prime \prime} 2=h^{2}-h^{\prime 2} \text {. }
\end{aligned}
$$

Here $h^{\prime \prime}$ is the true half-width, $h$ the observed half-width, and $h^{\prime}$ the half. width of the slit function. However, no single function of either type gives a good fit to both the observed line shape and the slit function. It has been found that the present experimental curves for the observed line profile and the slit function can be quite adequately fitted by Voigt functions (Voigt 1912). These functions are defined as the convolution integral between a gaussian and a lorentzian function. They may be used to fit any profile which lies between a gaussian and a lorentzian curve. If $f(x)$ and $f^{\prime}(x)$ are both taken to be Voigt functions, then the true line shape is also a Voigt function by Eq. (2-1). One may thus write observed line shape,

$$
f(x)=M \int_{-\infty}^{+\infty} \frac{\exp \cdot\left[-\left(y / \beta_{2}\right)^{2}\right]}{1+\left[(x-y) / \beta_{1}\right]^{2}} d y
$$

slit function,

$$
f^{\prime}(x)=M^{\prime} \int_{-\infty}^{+\infty} \frac{\exp \cdot\left[-\left(y / \beta_{2}^{\prime}\right)^{2}\right]}{1+\left[(x-y) / \beta_{1}^{\prime}\right]^{2}} d y ;
$$

true line shape,

$$
f^{\prime \prime}(x)=M^{\prime \prime} \int_{-\infty}^{+\infty} \frac{\exp \cdot\left[-\left(y / \beta_{2}^{\prime \prime}\right)^{2}\right]}{1+\left[(x-y) / \beta_{1}^{\prime \prime}\right]^{2}} d y ;
$$


where $M, M^{\prime}$, and $M^{\prime \prime}$ are constants. It is a property of these functions and Eq. (2-1) that

$$
\beta_{1}^{\prime \prime}=\beta_{1}-\beta_{1}^{\prime}
$$

and

$$
\beta_{2}^{\prime \prime} 2=\beta_{2}^{2}-\beta_{2}^{\prime} 2
$$

Figure 3 illustrates a typical Voigt analysis. In addition to the width $h$ at half maximum height, the width $b_{1}$ at one-tenth the height, $b_{2}$ at two-tenth the maximum height, etc., are measured. After calculating the ratios $b_{i} / h$, where $i$ goes from one to eight, Table 1 (van de Hulst and Reesinck 1947) was used to obtain the corresponding Voigt parameters $\beta_{1} / h$ and $\boldsymbol{\beta}_{2}{ }^{2} / h^{2}$, which were appropriately averaged over different $b_{i}$ for a given absorption line.

A lorentzian line shape gives $\beta_{1} / h=0.500, \beta_{2}{ }^{2} / h^{2}=0$; while a gaussian profile leads to $\beta_{1} / \mathrm{h}=0, \beta_{2}{ }^{2} / \mathrm{h}^{2}=0.36$. The atmospheric water vapor lines investigated to determine the slit function (Figure 2) were all found to lead to

$$
\beta_{1}^{\prime} / h^{\prime}=0.28 \pm 0.08 ; \beta_{2}^{\prime 2} / h^{\prime 2}=0.16 \pm 0.06 \text {. }
$$

Using the water vapor absorption line half-widths from Figure 2, Eq. (2-8) enables us to find the slit function voigt parameters $\beta_{1}^{\prime}$ and $\beta_{2}^{\prime}{ }^{2}$. Performing the analysis in the same fashion for the observed absorption lines in boron-doped silicon, one finds the Voigt parameters $\beta_{1}$ and $\beta_{2}$. One may then use Eq. $(2-6)$ and $(2-7)$ to obtain the Voigt parameters $\beta_{1}^{\prime \prime}, \beta_{2}^{\prime \prime 2}$ for the true absorption lines. Within experimental error $\beta_{2}^{\prime \prime 2}$ was found to be equal to zero, which means the Voigt functions for the true absorption lines simplify to lorentzian profiles. That is, Eq. (2-5) becomes 


\section{FIGURE 3.}

Typical Voigt analysis (water vapor line at $298.6 \mathrm{~cm}^{-1}$ ).

The numbers $b_{i} / h$ (where $i$ goes from 1 to 8 ) give the

line width at $i / 10$ of the maximum height, relative to the line width at half maximum height, (h).

The values $\beta_{1} / h$ where obtained from Table 1 . 


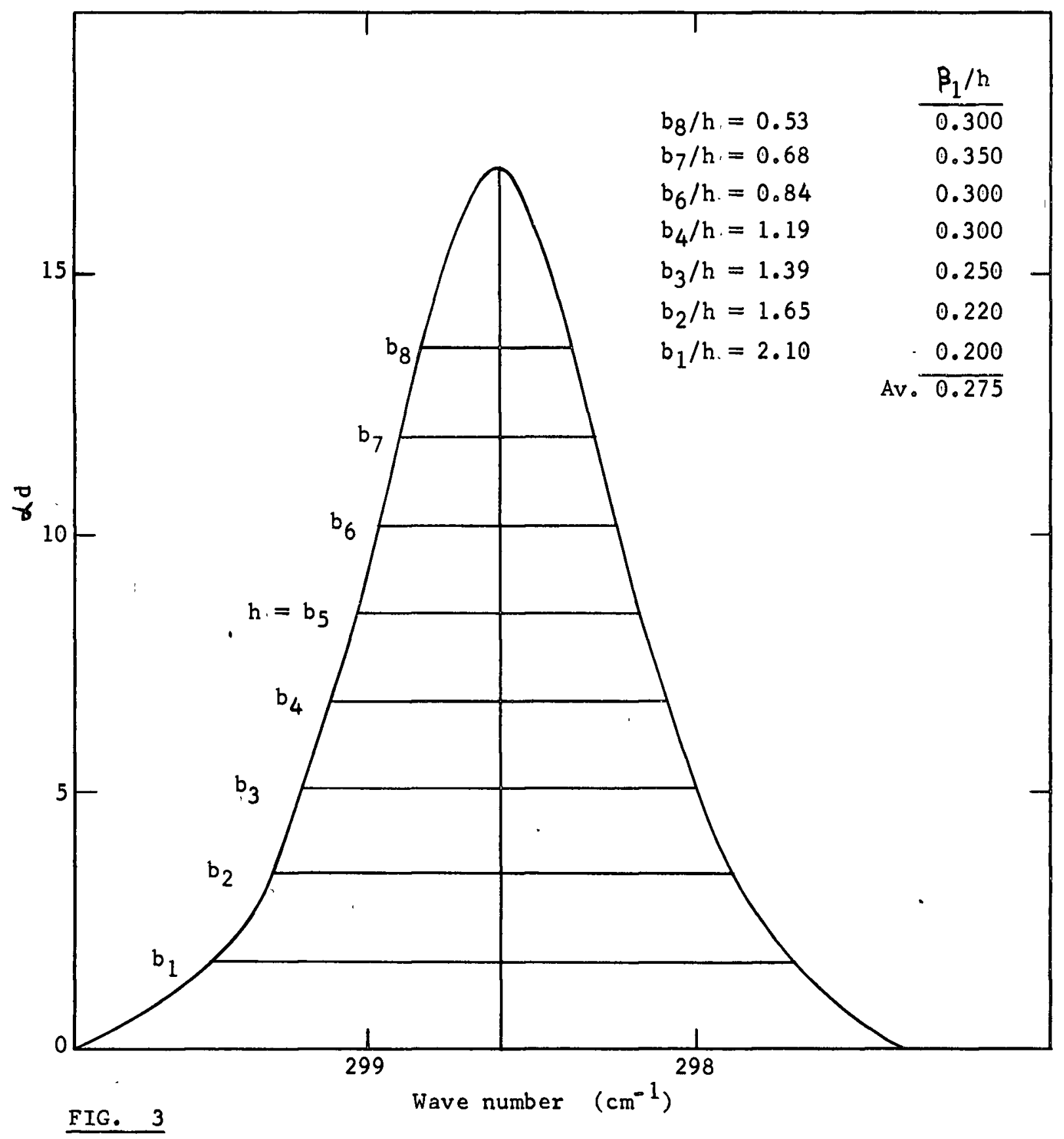




$$
f^{\prime \prime}(x)=c^{\prime \prime}\left(1+x^{2} / \beta_{1}^{\prime \prime 2}\right)^{-1},
$$

where $c^{\prime \prime}$ is the maximum height of the curve, occurring at $x=0$; and one has $\beta_{1}^{\prime \prime} / \mathrm{h} "=0.50$ 。

It was found that for the observed absorption lines in boron-doped silicon $\beta_{1} / h$ varied between 0.45 for narrow lines to about 0.5 for broad lines. Under these conditions, with $h^{\prime \prime}=2 \beta_{1}^{\prime \prime}, E q \cdot(2-6)$ leads to

$$
h^{\prime \prime}=g h-g^{\prime} h^{\prime},
$$

where $g=2 \beta_{1} / \mathrm{h}$ varies between 0.9 and 1.0 for different absorption lines, and by. Eq. $(2-8)$ one has $g^{\prime}=2 \beta_{1}^{\prime} / h^{\prime}=0.56 \pm 0.16$.

The values listed in Table 1 under $p$ may be used to evaluate the integrated absorption $A$. Both $A$ and $p$ are defined by

$$
A=\int_{-\infty}^{+\infty} f(x) d x=p h c
$$

for the observed line, or similarly for the true line by

$$
A^{\prime \prime}=\int_{-\infty}^{+\infty} f^{\prime \prime}(x) d x=p^{\prime \prime} h^{\prime \prime} c^{\prime \prime},
$$

where $c$ and $c$ " are respectively the observed and the true central ordinate (maximum height). Since the integrated absorption is not changed by spectrometer broadening provided one scans over a reasonably wide wave number range, one has

$$
\text { p h } c=p^{\prime \prime} h^{\prime \prime} c^{\prime \prime} \text {. }
$$

Knowing the observed integrated absorption, $h^{\prime \prime}$, and $\beta_{1}^{\prime \prime} / h^{\prime \prime}, E q \circ(2-13)$ and Table 1 enables one to find the true central ordinate $c^{\prime \prime}$, if desired. With the present experimental data it is a good enough approximation to take in Eqs. (2-11), (2-12), and (2.13) from Table 1

$$
\mathrm{p} \approx \mathrm{p}^{\prime \prime}=1.54 \pm 0.03
$$

for all absorption lines investigated. 


\section{Results and Discussion of Errors}

Correcting the data in Figure 1 for spectrometer distortion by the methods outlined in the previous section, the true halfowidths $h^{\prime \prime}$ were obtained. These have been plotted in Figure 5 as a function of temperature for three concentrations of boron impurities. In Figure 4 these data are extrapolated to zero impurity concentration. The resulting curves are shown in Figure 5 as dotted lines. For the purpose of extrapolation a plot of $\ln h^{\prime \prime} \mathrm{vs}$. $\mathrm{N}_{\mathrm{A}}^{1 / 3}$ was found convenient and results in reasonably straight lines。

The main contribution to the uncertainty in $h^{\prime \prime}$ (Figure 5) has varying origins for different temperatures and lines under study. This will partly be illustrated by reference to Figure 7 , which shows in magnified form the absorption constant of 1 ines 3 and 4 in 11 ohm $\mathrm{cm}$ boronodoped silicon at four different temperatures. For rather sharp lines (low temperature and impurity concentration) the main source of error comes from the uncertainty of $g$ and $g^{\prime}$ in Eq. (2-10), together with the fact that one subtracts two similar numbers. The extreme case for this is line 1 in $130 \mathrm{ohm} \mathrm{cm}$ at $4.2^{\circ} \mathrm{K}$ 。 Here $\mathrm{h}=0.153 \mathrm{mev}, \mathrm{h}^{\prime}=0.095 \mathrm{mev}$, and using Eq. $(2-10)$ one obtains $h^{\prime \prime}=0.08 \pm 0.04 \cdot \mathrm{mev}$. On the opposite extreme, for wide lines (high temperature and impurity concentration) the uncertainty in the half-width results primarily from the difficulty in peelingooff the absorption due to neighboring lines.

Two other sources of uncertainty are important only for special cases. The first is the rather large absorption in the $1.3 \mathrm{ohm} \mathrm{cm}$ material, which gives rise to an uncertainty of about five percent in the peak-heights of 1 ines 2 and 4 at $4.2^{\circ} \mathrm{K}$ and $60^{\circ} \mathrm{K}$. The second source of error is the presence of some background absorption lines due to atmospheric water vapor. 


\section{FIGURE 4.}

Extrapolation of the true half-width (logarithmic scale)

to zero concentration of boron impurities $\left(\mathrm{N}_{\mathrm{A}}\right)$.

To follow page 12 . 


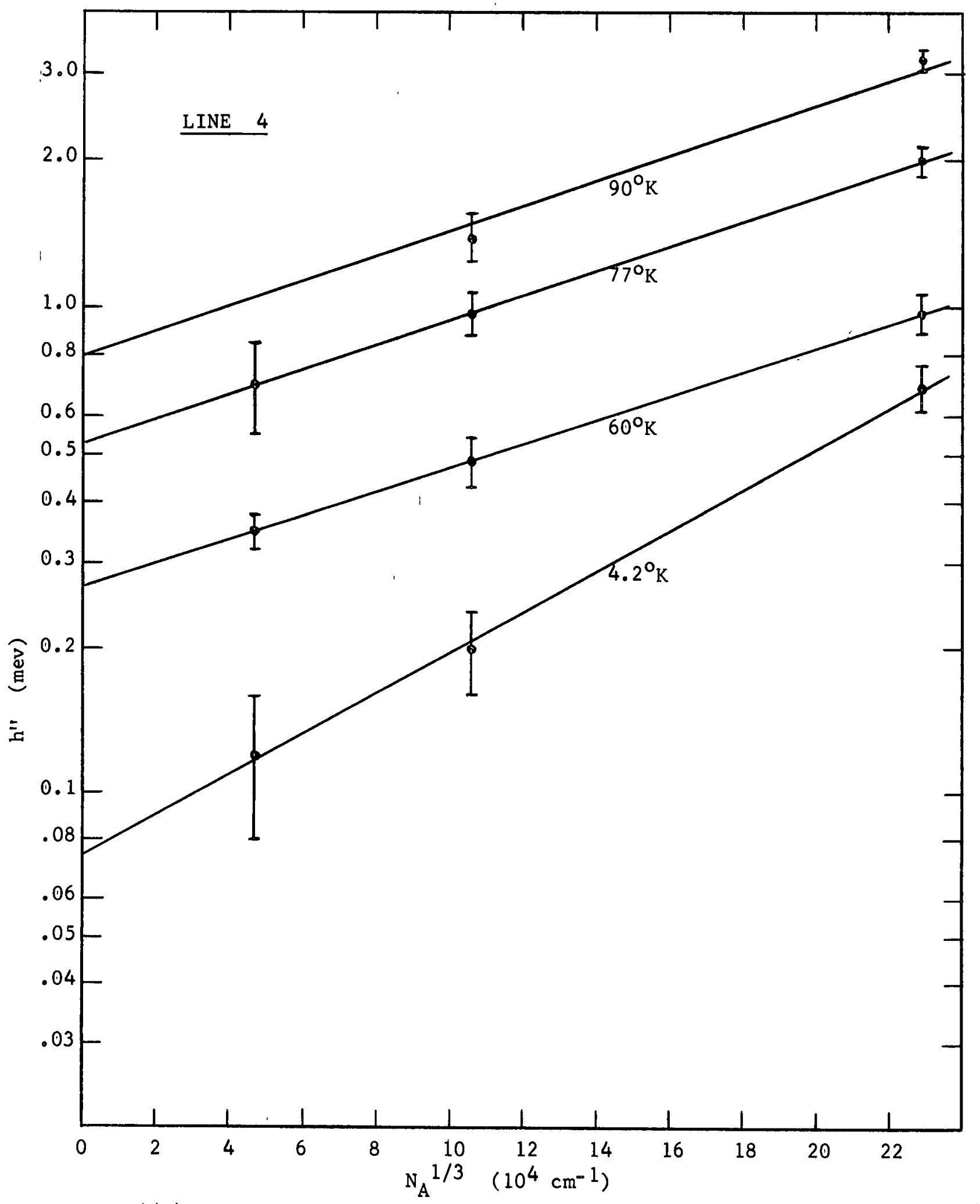

FIG. $4(a)$ 


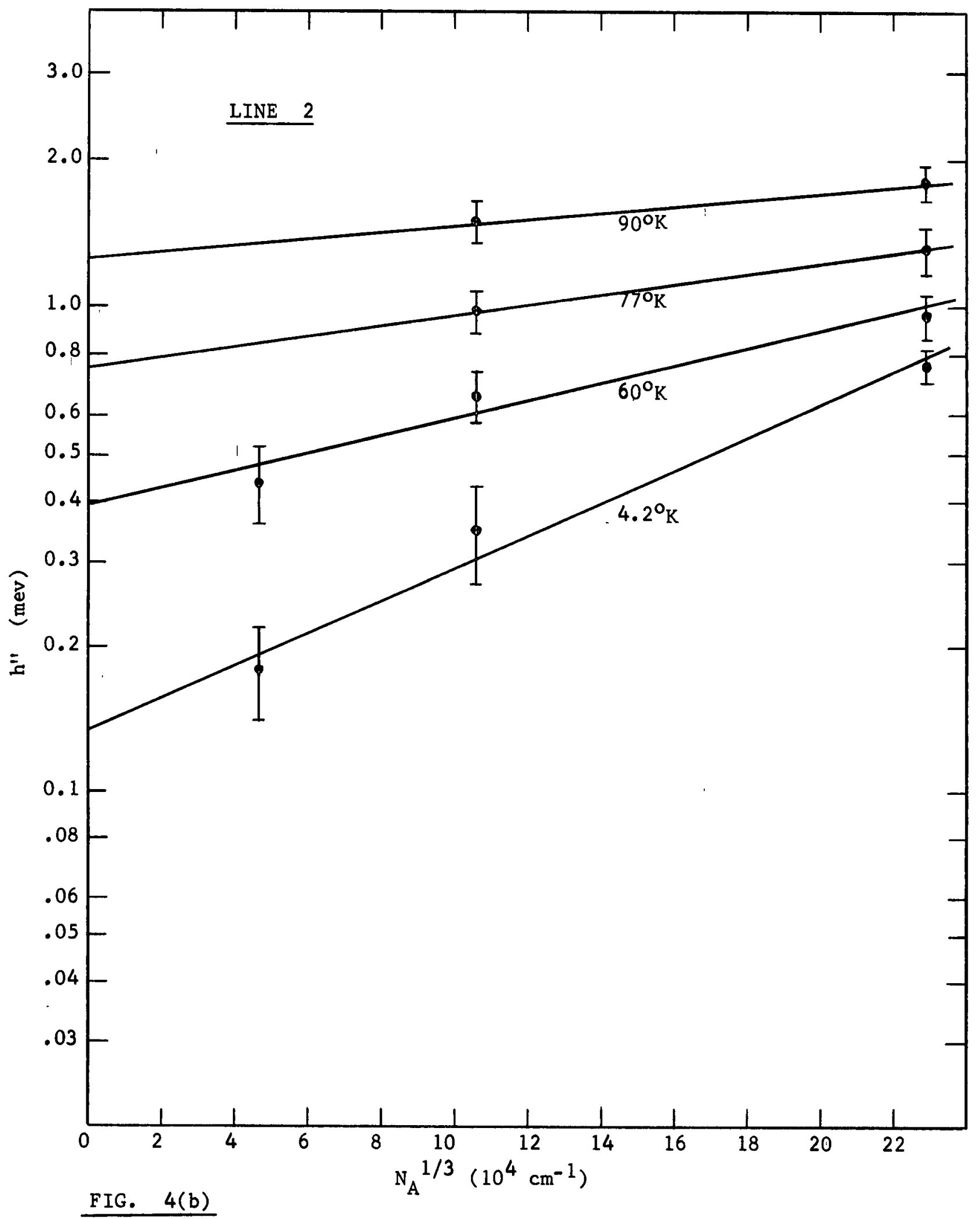




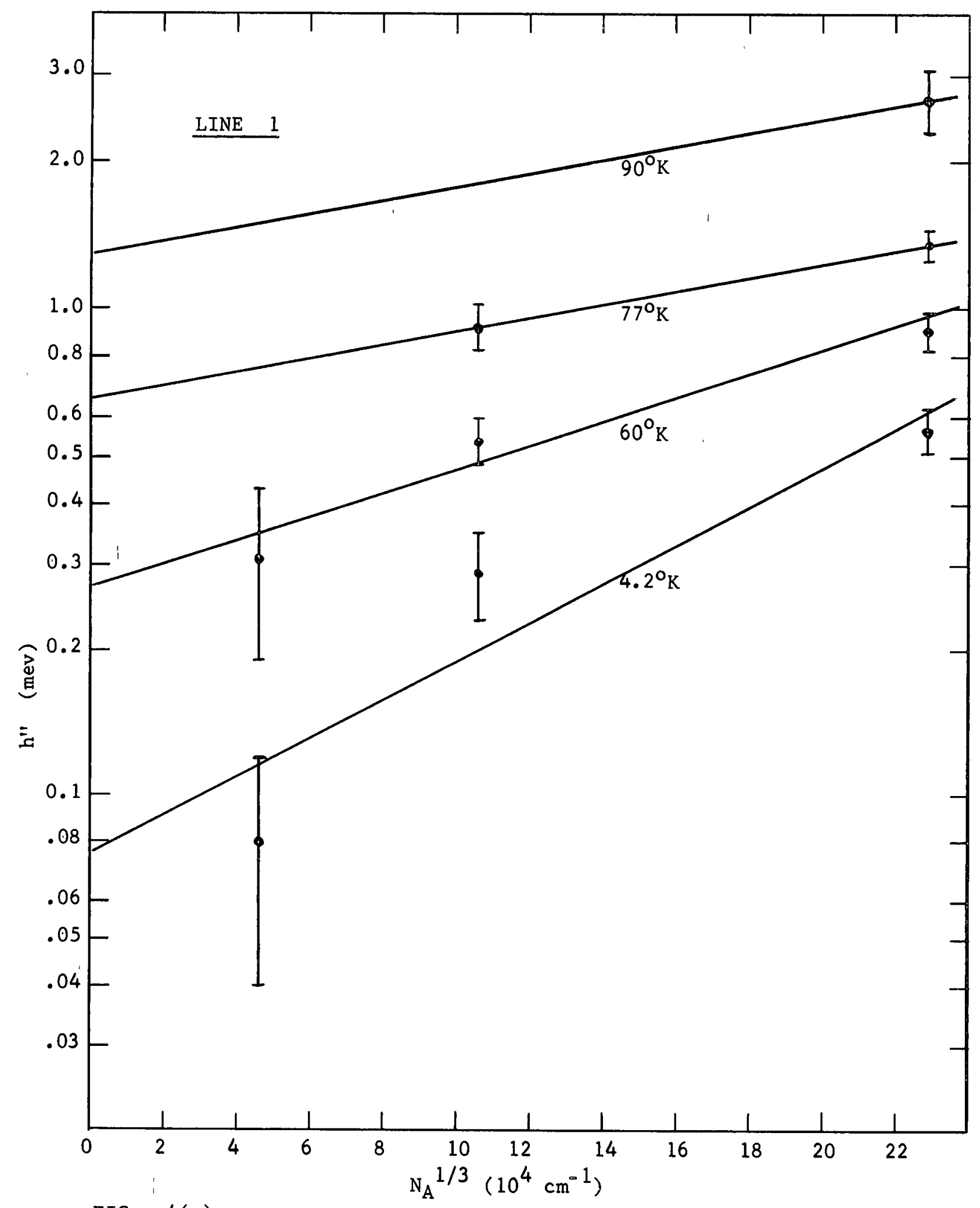

FIG. $\quad 4(c)$ 


\section{FIGURE 5.}

True half-width (corrected for spectrometer broadening) vs.

temperature for boron concentrations of i) $1.0 \times 10^{14} \mathrm{~cm}^{-3}$,

ii) $1.2 \times 10^{15} \mathrm{~cm}^{-3}$, iii) $1.2 \times 10^{16} \mathrm{~cm}^{-3}$, and iv) very

low concentrations (extrapolated). 


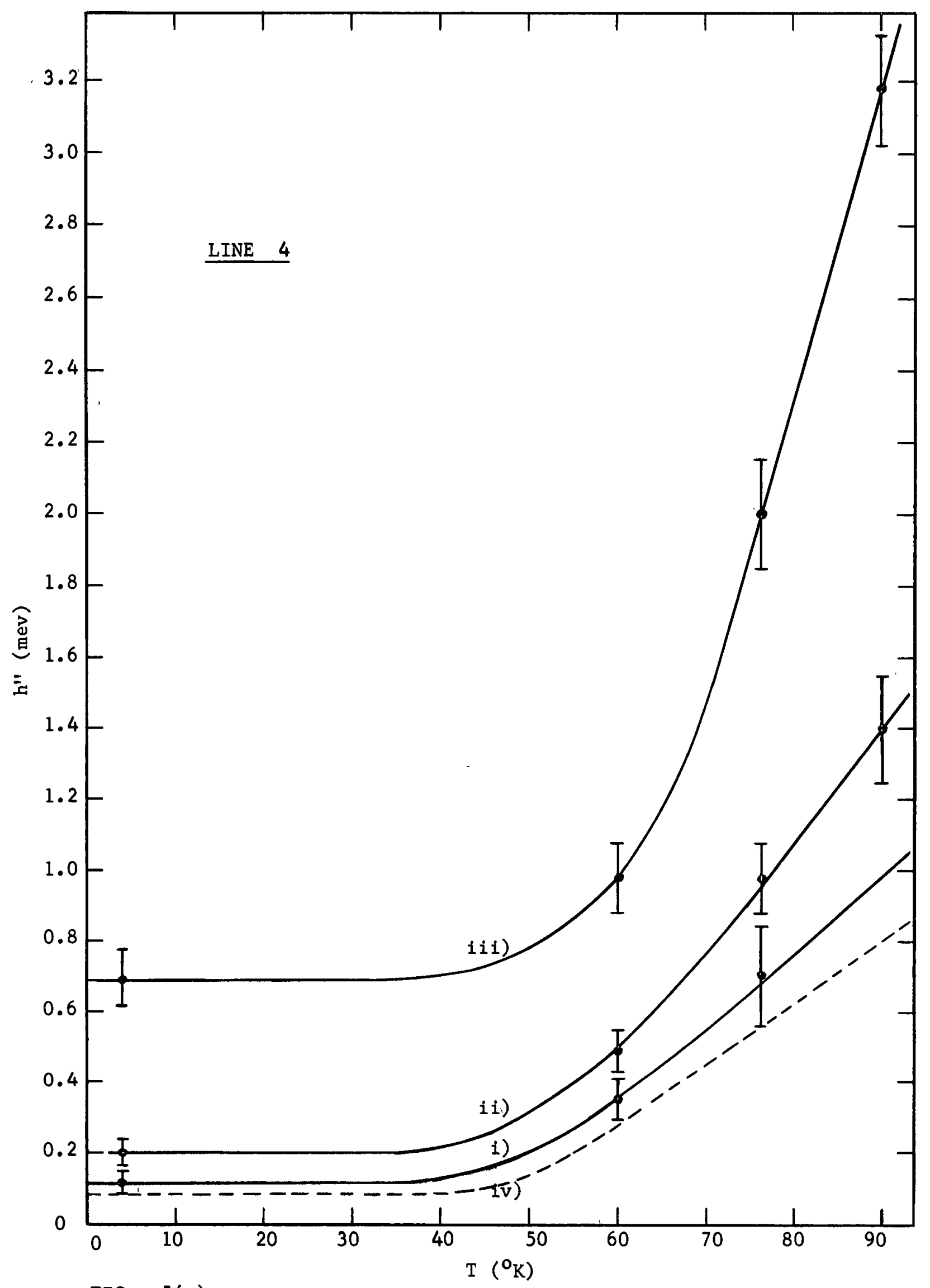

FIG. 5(a) 


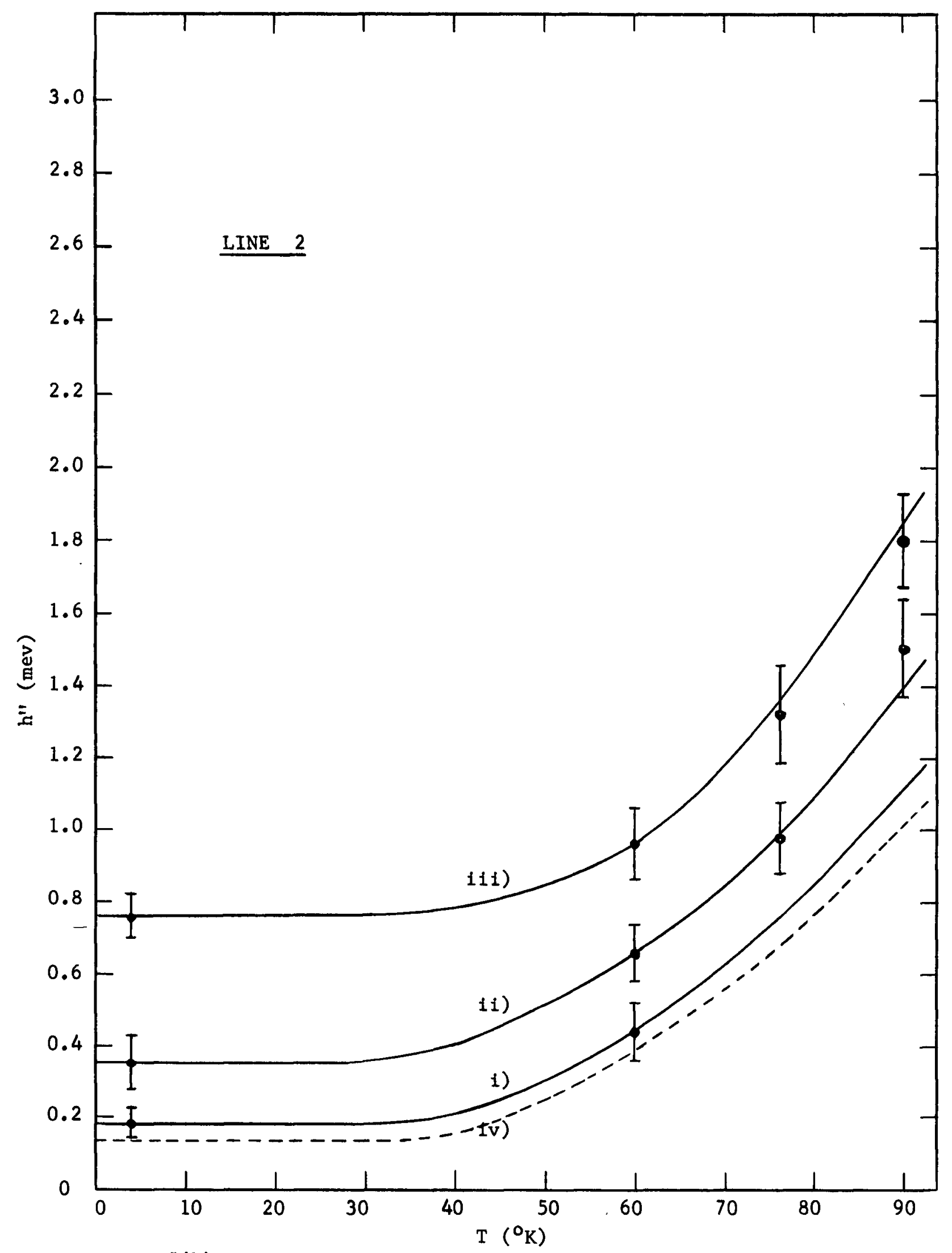

FIG. $5(\mathrm{~b})$ 


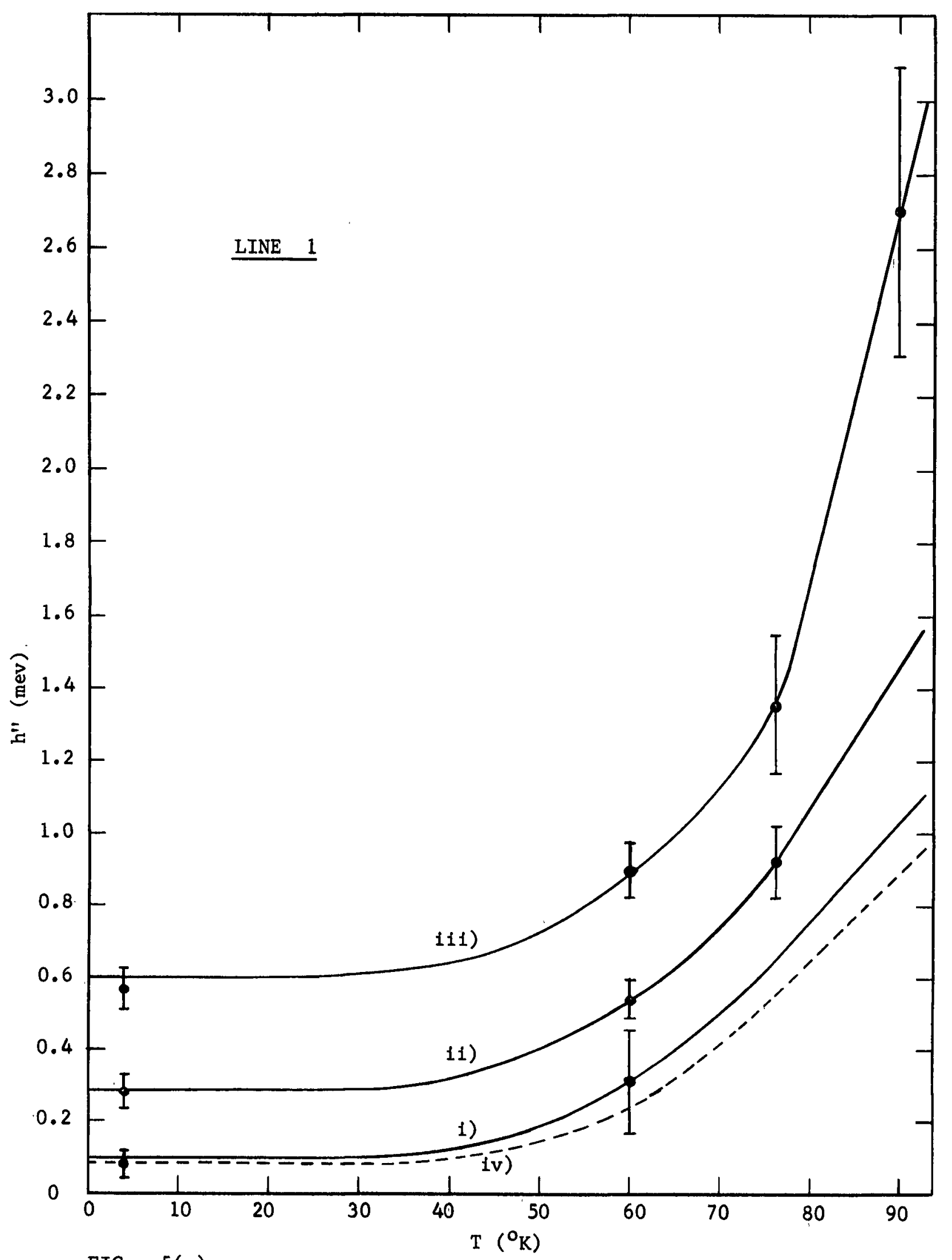

FIG. $\quad 5(c)$ 


\section{FIGURE 6.}

Half-width minus zero-concentration half-width (extrapolated) vs. temperature for line 4. 


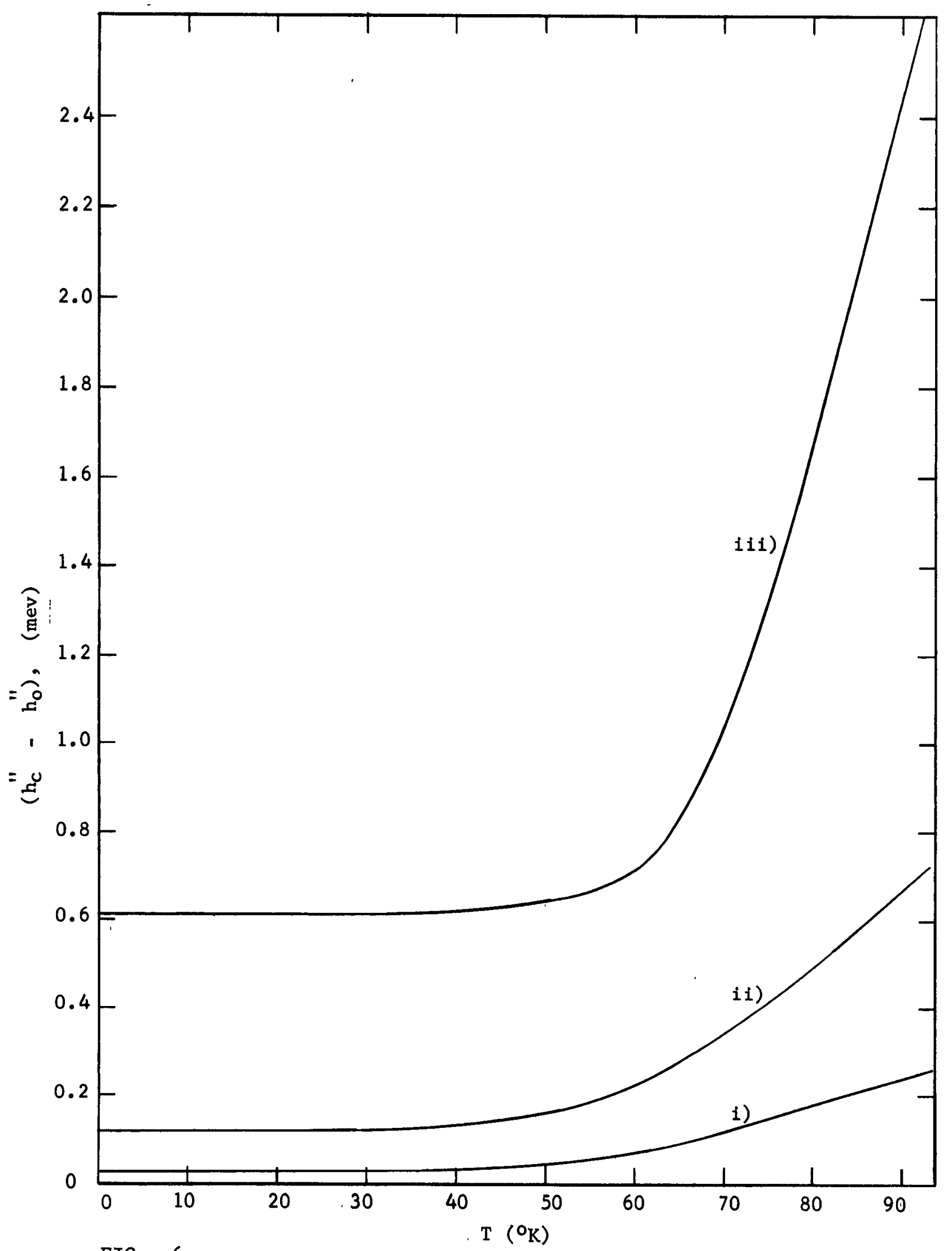

FIG. 6 


\section{EIGURE 7.}

Absorption constant vs, wave number for $11 \mathrm{ohm} \mathrm{cm}$

$\left(1.2 \times 10^{15} \mathrm{~cm}^{-3}\right)$ boron-doped silicon at four

temperatures. This figure illustrates some of the

difficulties and uncertainties involved in "peeling"

off the influence of neighboring 1 ines.

To follow page 12 . 

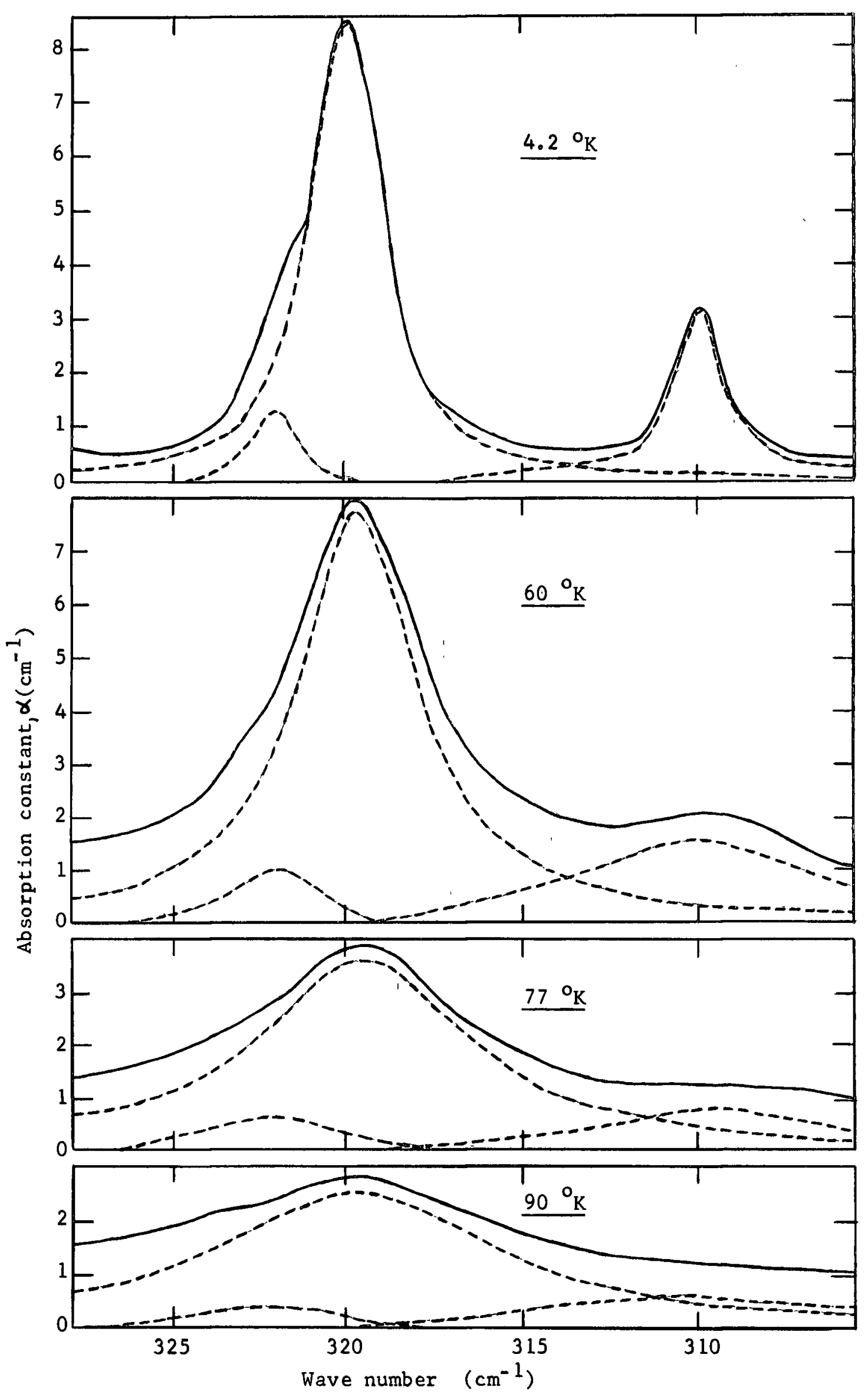

FIG. 7 
Incomplete cancellation of water vapor lines may be responsible for the small bump on the high energy side of line 4 (see Fig.7). Similarly, the dip in line 2 reported earlier (Colbow et al. 1962) may be due to water vapor. The fact that a rather strong water vapor absorption line occurs right at the position of line 2 gives the data for this line (Figure 5b) less reliability than those for 1 ine 4 and 1 (Figures $5 a, c$ ). Since a more complete set of half-width measurements was accessible for line 4, Figure 5 a will be used exclusively in the quantitative interpretations in Chapter III.

Other pieces of information extracted from the data in Figure 1 are the positions of the absorption lines (Figure 8) and the integrated absorption (Table 2)。The energy levels in Figure 8 have been plotted by assuming an ionization energy of $46 \mathrm{mev}$ (Burstein et al。1956, Kohn 19.57)。 The present author believes that an ionization energy of possibly as low as 44 mev cannot be ruled out by the present experimental data. This value would give better agreement between the experimental and the calculated (Schechter 1962) positions of excited states (Figure 8)。

The experimentally determined positions of the absorption lines are essentially in agreement with those obtained earlier by Hrostowski and Kaiser $(1958)$. At $4.2^{\circ} \mathrm{K}$ an additional line was resolved near the ionization edge in the $130 \mathrm{ohm} \mathrm{cm}$ material。

The uncertainty of the integrated absorption in Table 2 is about 10 percent for all lines from the uncertainty in the product $p h c$, i.e. Eq. (2-11), In addition an uncertainty of approximately 5 percent exists due to the fact that the wings of the absorption lines may not be well fitted by Voigt profiles. However, this is difficult to estimate due to an overlap of neighboring lines. 
The integrated absorption crossosections $(\Sigma)$ in Table 3 were obtained by dividing the integrated absorption (Table 2) by the rumber of boron impurities in the ground state (Figure 9). The uncertainty of $\Sigma$ is estimated to be approximately 25 percent. 


\section{FIGURE 8.}

Energy level diagram for holes in boron-doped silicon. The "experimental" energy level diagram (left side) was obtained by taking the ground state at -46 mev. Along the vertical arrows the positions of the observed absorption lines are given in mev and $\mathrm{cm}^{-1}$. Thẹ numbers in the center of the diagram ( 1 to 9 ) serve to identify the levels for discussion in this thesis. The relative intensities quoted in the diagram refer to a boronconcentration of $1.0 \times 10^{14} \mathrm{~cm}^{-3}$ at $4.2{ }^{\circ} \mathrm{K}$. For comparison, the right side of Figure 8 shows the calculated positions (Schechter 1962) for the ground state and the lowest four excited states. The numbers in brackets give the theoretical degeneracies. 


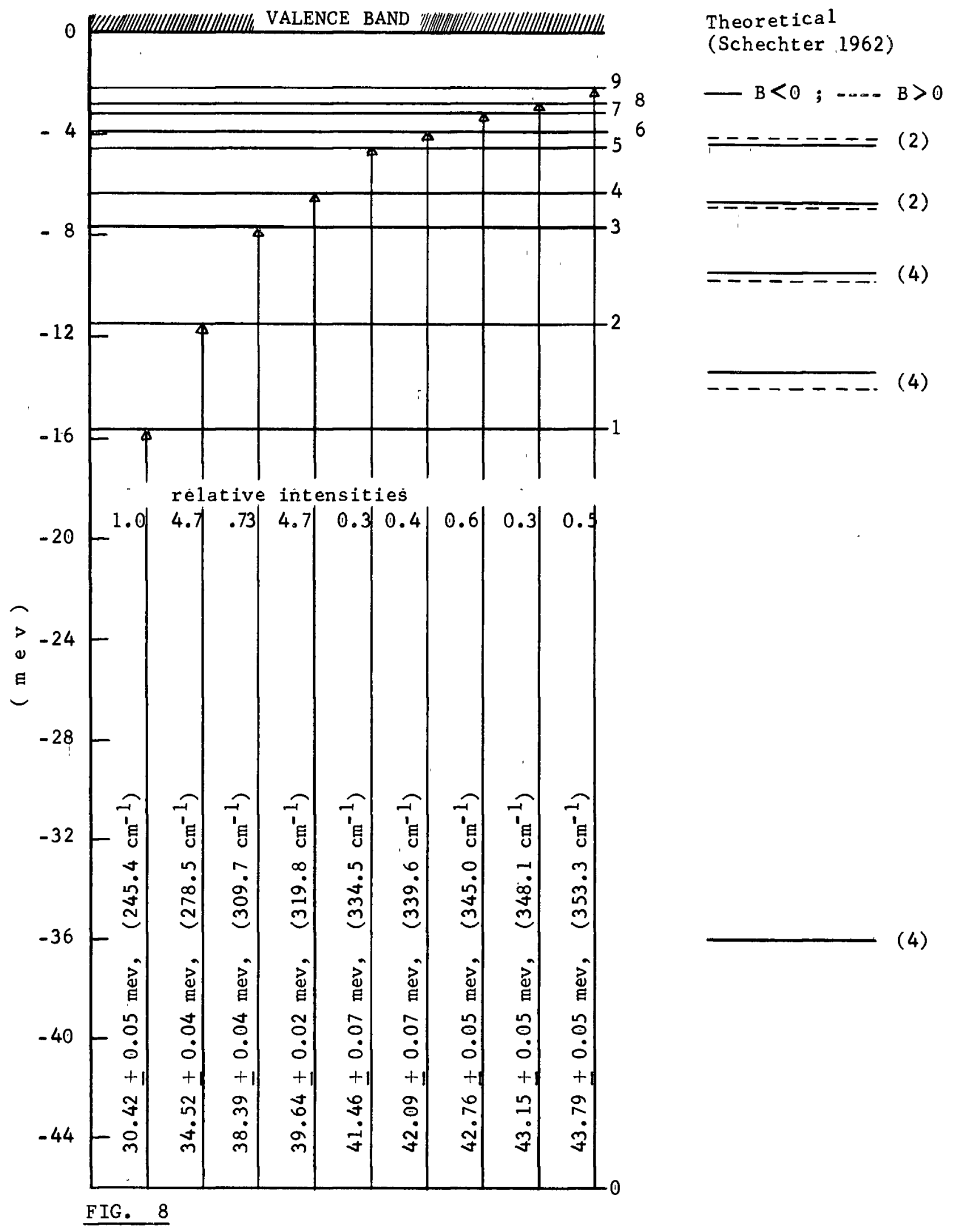




\section{FIGURE 9.}

Distribution of holes between the ground state $(\underset{i}{a})$, excited states (b), and the valence band (c) vs. temperature. The boron concentrations are: 1) $1.0 \times 10^{14} \mathrm{~cm}^{-3}$,

2) $1.2 \times 10^{15} \mathrm{~cm}^{-3}$, and 3) $1.2 \times 10^{16} \mathrm{~cm}^{-3}$. The ordinate gives the number of holes in units of $10^{14}, 10^{15}$, and $10^{16}$ $\mathrm{cm}^{-3}$ for cases 1 ), 2), and 3) respectively. 


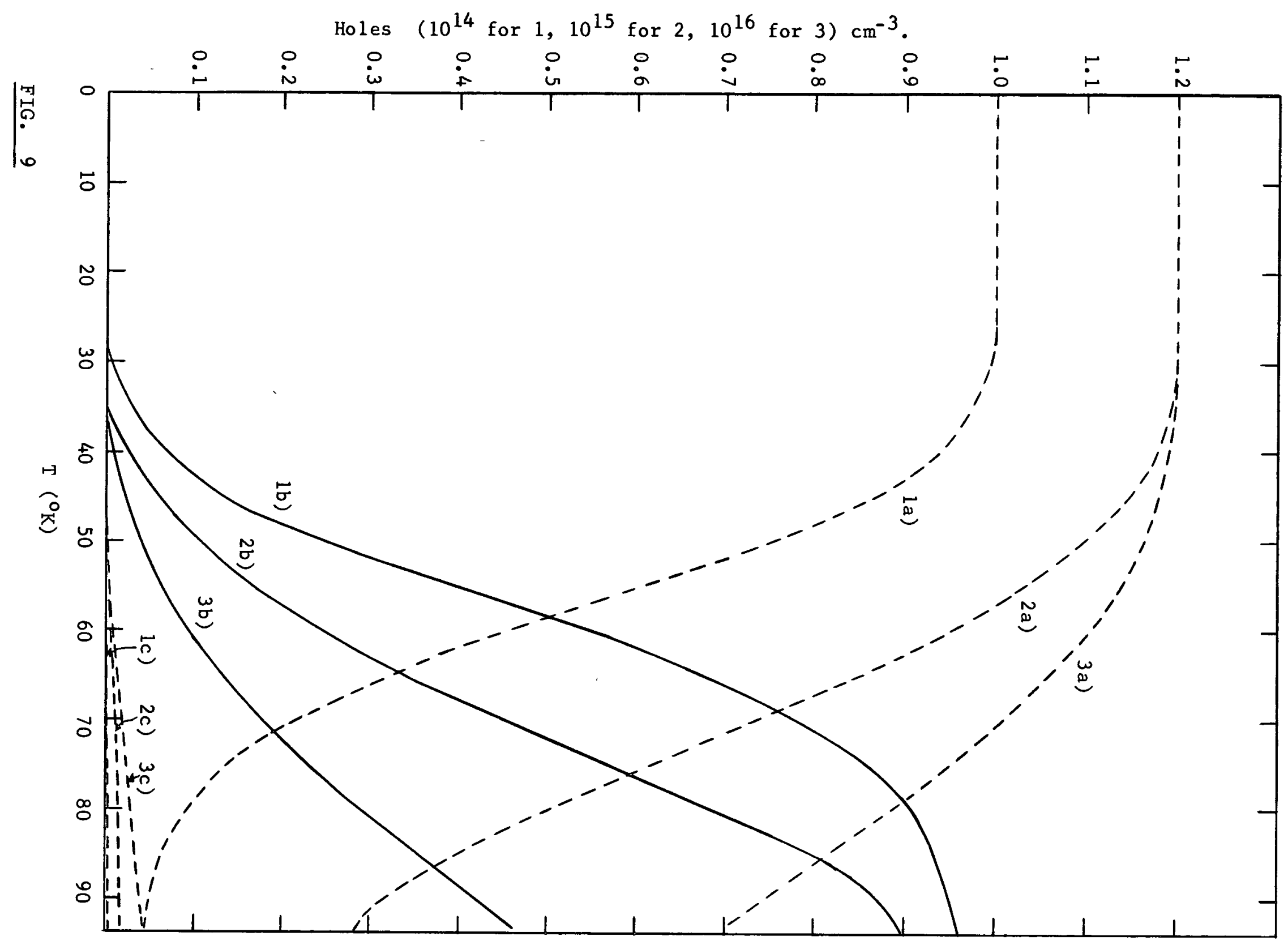




\section{CHAPTER III}

\section{Impurity States and the Hydrogenic Approximation}

Silicon is a group IV semiconductor, and its electronic structure is such that at zero temperature the electrons fill the valence band, leaving the conduction band empty. When a small quantity of a group III or a group $\mathrm{V}$ element is added, a set of energy levels, localized at the impurity atom is formed. For group $\mathrm{V}$ impurities these are referred to as donor levels. They correspond to an electron loosely bound to the impurity ion. This electron can be "donated" to the conduction band with a small expenditure of energy. In the case of group III elements the levels are referred to as acceptor levels, since they can accept an electron from the valence band. Alternatively one may picture acceptor levels as containing holes loosely bound to the impurity ion. Boron is a group III element and gives rise to acceptor levels in silicon.

At room temperature the electrons and holes are removed from the impurity sites by thermal excitations, and exist as free charge carriers in the crystal, while at liquid helium temperature nearly all these carriers are bound to specific impurity sites and occupy the lowest energy state。 These bound states have been extensively studied (Kohn 1957), and it has been shown that they can be approximately, described by the same schrodinger equation as the states of an electron in a hydrogen atom, but with the Coulomb potential modified by the static dielectric constant $K$, and the electron mass replaced by an effective mass $\mathrm{m}^{*}$, (Kittel and Mitchel1 1954, Luttinger and Kohn 1955):

$$
\left(-2 / 2 m^{*} \nabla^{2}-e^{2} / K r\right) F\left(\vec{r}^{*}\right)=E F(\vec{r}) \text { 。 }
$$

In analogy with the solutions of the hydrogen atom, one expects an energy level scheme with the energies reduced by $\mathrm{m}^{*} / \mathrm{m} \mathrm{K}^{2}$ and the Bohr orbits 
enlarged by $\mathrm{Km} / \mathrm{m}^{*}$. Taking for boron-doped silicon $\mathrm{K}=12$ and $\mathrm{m}^{*} / \mathrm{m}=0.5$ one obtains roughly the observed value for the ionization energy of $46 \mathrm{mev}$ 。 The radius of the first Bohr orbit turns out to be $13 \stackrel{\circ}{\mathrm{A}}$ for acceptors, rather than the $0.53 \mathrm{~A}$ in hydrogen. These large Bohr orbits give an explanation of why such a simple description of impurity states is possible. The mean distance between the bound carrier and the impurity is sufficiently large for a macroscopic quantity like the dielectric constant to be useful. Also, the orbits of the bound carriers are sufficiently large that we need not be concerned with the detailed nature of the wave function right at the impurity site. This in any case is true of excited states, such as $2 \mathrm{p}$ states, whose wavefunctions vanish at the impurity site. 'The sostates are likely to be much more complicated and depressed in energy, since the eigenfunctions are large right at the impurity site where the potential is no longer modified by the dielectric constant.

This description of impurity states would be quite good, if the energy vs。 wave number vector diagram were of parabolic shape and had a simple extremumat $k=0$. The wave functions of the acceptor states are linear combinations of Bloch waves chosen from near the top of the valence band, and the effective mass schrödinger equation (1-1) corresponds simply to an expansion of the energy around $k=0$, keeping terms to order $k^{2}$. The fact that we are concerned only with small $k$ follows from the uncertainty principle. The large spatial extension of the Bohr orbit implies a small extension for the crystal momentum $k$.

Unfortunately, the valence band in silicon is not simple. Information about the structure of this band has been obtained from cyclotron resonance exper iments by Lax, Zeiger, and Dexter (1954) and Dresselhaus, Kip, and Kittel (1955). If it were not for spin-orbit coupling the valence band maximum at $k=0$ would be sixfold degenerate, 
including the double degeneracy arising from spin. The simples way to understand this degeneracy is to consider the tight binding limit, in which the wave functions corresponding to the maximum go over into $3 p$ atomic wave functions for silicon. The spin-orbit coupling lifts the degeneracy partially. According to Elliott (1954) the top of the valence band remains fourfold degenerate, corresponding to atomic $\mathrm{J}=3 / 2$ states, splitting away from $k=0$ in the (100) direction into twofold degenerate bands. Another twofold degenerate state lies an amount $\lambda$ below the top of the valence band corresponding to $\mathrm{J}=1 / 2$. A theoretical estimate (Kohn 1957) gives $\lambda=35 \mathrm{mev}$.

Since in boron-doped silicon the highest acceptor states (lowest energy states for bound holes) lie above the valence band by about $40 \mathrm{mev}$, one expects that all six valence bands will enter with appreciable amplitude in the acceptor states. Instead of a single partial differential equation, Eq. (1-1), one finds a set of six coupled partial differential equations. These have been derived by Kittel and Mitchell (1954) and Luttinger and Kohn (1955). They are

$$
\begin{aligned}
& \sum_{j=1}^{6}\left\{D_{j}^{\alpha} j_{j}^{\beta}\left(-\frac{1}{i} \frac{\partial}{\partial x_{\alpha}}\right)\left(\frac{1}{i} \frac{\partial}{\partial x_{\beta}}\right)-\left\{\begin{array}{c}
2 / X r \\
-\lambda \sigma_{j}
\end{array}\right\} \delta_{j j}, F_{j,}(\vec{r})=0 .\right. \\
& \sigma_{j}=\left\{\begin{array}{lll}
0 & \text { for } j=1,2,3,4 . \\
1 & \text { for } j=5,6 .
\end{array}\right.
\end{aligned}
$$

Here $\epsilon$ is an acceptor state energy relative to the valence band maximum. The $D_{j}^{\alpha} \beta_{j}$, are numbers related to three effective mass constants $A, B, C$ (which are determined from cyclotron resonance data, and fromthe spin-orbit splitting $\lambda$ ). The specific form of the $D_{j}^{\alpha} \beta$, may be found in the articles by Kittel and Mitchel1 (1954) and Luttinger and Kohn (1955).

In terms of the effective mass constants, the energies of the three valence bands are given by 


$$
\begin{gathered}
E_{1,2}(\vec{k})=A k^{2}:\left[B^{2} k^{4}+C^{2}\left(k_{x}{ }^{2} k_{y}{ }^{2}+k_{y}{ }^{2} k_{z}{ }^{2}+k_{z}{ }^{2} k_{x}{ }^{2}\right)\right]^{1 / 2}, \\
E_{3}(\vec{k})=-\lambda+A^{2},
\end{gathered}
$$

each eigenvalue being doubly, degenerate.

The total wave functions of these acceptor states have the form

$$
\begin{aligned}
\psi(\vec{r}) & =\sum_{n, k} A_{n}(\vec{k}) \psi_{n, \vec{k}} \\
& \approx \sum_{j=1}^{6} F_{j}(\vec{r}) \quad \phi_{j}(\vec{r}) .
\end{aligned}
$$

Here the $\phi_{j}(\vec{r})$ are the Bloch functions at the top of the valence bands in the unperturbed crystal. The $F_{j}(\vec{r})$ are slowly varying functions which modulate the Bloch functions. For this reason they are often referred to as envelope functions. In the limit where the mass constants $B$ and $C$ become zero (corresponding to spherical constant energy surfaces), the envelope functions reduce to simple hydrogenic functions. Schechter (1962) attempted to solve Eq. (1-2). In order to simplify the calculations he first calculated the energy levels in the extreme limits of $\lambda=0$ and $\lambda=\infty$. First-order perturbation calculations were then made, in $\lambda$ and $\lambda^{-1}$ in order to obtain estimates for the energy levels corresponding to the actual, finite value of $\lambda$. Expanding the envelope functions $F_{j}(\vec{r})$ in spherical harmonics, Schechter's acceptor state trial functions had the form

$$
\psi=\sum_{1} r^{1} \exp \left(-r / r_{1}\right) \sum_{j, m} c_{1 m}^{j} Y_{1 m}(\theta, \phi) \phi_{j}(\vec{r}) .
$$

The complete acceptor Hamiltonian is invariant under the operations of the full tetrahedral group $T_{d}$ (Margenau and Murphy 1943) if spin is ignored, or, the tetrahedral double group (Dresselhaus 1955) if spin is included from the beginning. The acceptor state wave functions 
for each level form bases for irreducible representations of the appropriate symmetry group. Since the spherical harmonics for each 1 go into spherical harmonics of the same 1 under the symmetry operations, each term in the sum over 1 in Eq.(1-5) must transform in the same way as the complete wave function. After using group theoretical arguments to reduce the number of spherical harmonics appearing in the trial functions, and restricting 1 to values less than some $1_{0}$, the best approximations to the wave function and the energy were obtained by maximizing the expectation value of the energy for an electron state. (This corresponds to minimizing the energy for a hole state).

The sign of the effective mass constant B is experimentally not determined, since the valence band energies depend only on $B^{2}$ by Eq. (1-3). However, B occurs linearly in the $D \underset{j}{\alpha} j^{\prime}$ of Eq. (1-2). Schechter (1962) calculated the ground state energy and those of the highest four excited electron acceptor states for both positive and negative B. The positions of these calculated levels, together with their degeneracies (in brackets) are shown in Fig. 8. The agreement with experiment is seen to be only fair. The levels are either fourfold degenerate corresponding to the representation $\Gamma_{8}$, or twofold corresponding to $\Gamma_{6}$ and $\Gamma_{7}$ of the tetrahedral double group. The ground state is predominantly $1 \mathrm{~s}-1$ ike and the lowest four excited states $2 \mathrm{p}$ - 1ike in the sense that they reduce to hydrogenic $1 \mathrm{~s}$ and $2 \mathrm{p}$ states respectively, in the limit when all three valence bands collapse into one.

In the following sections these rather complicated wave functions will not be used. Instead simple hydrogenic wave functions will be arbitrarily employed in which the first Bohr orbit is replaced by an effective Bohr orbit $a^{*}$. It will be required that this effective 
Bohr orbit satisfy the hydrogenic energy equation

$$
E_{n}=-e^{2} / 2 K a^{*} n^{2} \text {, }
$$

where $E_{n}$ is the experimental binding energy.

\section{Genera1 Interpretation of Line Broadening}

Consider a semiconductor with a random distribution of $\mathrm{N}_{1}$ ionized and $\mathrm{N}_{\mathrm{A}}-\mathrm{N}_{1}$ neutral acceptor or donor impurities. At an absorbing neutral impurity there will be a certain electric field, the magnitude of which depends on the specific spatial configuration of the surrounding impurities. This electric field may give rise to a first order Stark splitting, or second order Stark. shift, of different magnitudes for different impurity sites. Thus one obtains a broadening of the resultant absorption line. Let us suppose the distribution of intensity of absorption for one neutral impurity, which is in an electric field $F$, is given by I $(F, \omega)$; then in order to obtain the total intensity distribution in the Stark broadened line one must integrate over all such distributions weighted by the probability of the distribution occurrence:

$$
\text { I }(\Delta \omega) \cdot d \omega=d \omega \int_{0}^{\infty} I(F, \omega) W(F) d F \text {. }
$$

Here $W(F)$ is the field strength probability function, which has been studied by Holtsmark $(1919,1924)$ and others in connection with gravitational problems and pressure broadening in gases. This probability function will be discussed in the following section.

Each component of the statistically broadened absorption line will be treated as a sharp line, that is to say the $I(F, \omega)$ in Eq.(2-1) will be replaced by a delta function. Actually the true width of $I(F, \omega)$ may be obtained by extrapolating the experimental true width in Figure: 5, to zero concentration of impurities (dotted lines). These widths are seen to be 
already quite considerable, and are believed to result primarily from phonon broadening, and broadening by internal strains due to dislocations. The latter two broadening mechanisms will be discussed in sections 10 and 9 respectively.

After the width resulting from statistical stark broadening of two sharp energy levels has been obtained it has to be combined with the width at zero concentration by a convolution integral of the form

$$
f_{c}^{\prime \prime}(x)=\int_{-\infty}^{+\infty} f_{o}^{\prime \prime}(x-y) I(y) d y,
$$

where $f_{c}^{\prime \prime}(x)$ is the true line shape at concentration $c, f_{0}^{\prime \prime}(x-y)$ is the true line shape extrapolated to zero concentration of impurities, and $I(y)$ is the line shape obtained from statistical stark broadening under the assumption that the $I(F, \omega)$ in Eq. (2-1) are sharp lines. If one makes the simplifying assumption that $f_{c}^{\prime \prime}, f_{o}^{\prime \prime}$, and $I(y)$ have lorentzian profiles, then the half-width of $f_{c}^{\prime \prime}$ is simply the sum of the half-widths of $f_{0}^{\prime \prime}$ and $I(y)$. This assumption is at least approximately justified (Colbow et a1. 1962).

\section{Statistical Theory of Stark Broadening}

The basic question is, "What is the probability that the frequency of a sharp absorption line is displaced by an amount between $\Delta \omega$ and $\Delta \omega+\mathrm{d} \Delta \boldsymbol{\omega}^{\prime \prime}$ ? Let us assume that this frequency displacement results from the displacement of only one of the two levels involved in the absorption, by means of either the linear or the quadratic Stark effect. This will be justified later.

The probability of a certain frequency displacement due the surrounding impurities is then simply related to the probability of a certain electric field at the absorbing impurity. This in turn depends on 
the probability of finding another impurity at a distance $r$ from. it. Since the $I(F, \omega)$ in Eq. $(2-1)$ is taken to be a delta function, the probability. function for a frequency displacement between $\Delta \omega$ and $\Delta \omega+d \Delta \omega$ will give directly the desired line shape of the absorption line $I(\Delta \omega)$.

The problem may be divided into several parts according to the dependence of the field on the distance from the field producer. In the present section it will be assumed that the field at the absorbing neutral impurity results mainly from the surrounding ionized impurities, and has the form of a Coulomb field: $F=(e / 4 \pi \epsilon) r^{-2}$. This case becomes important above $40^{\circ} \mathrm{K}$, when an increasing number of impurities will be ionized (Figure 9). (In the following section the effect of screening by free holes will be discussed in an approximate fashion, and in sections 7 and 8 line broadening resulting from the interaction with neutral impurities will be considered). For the purpose of illustration it is useful to consider first the effect of only the nearest neighbor. This approximation may be referred to as the binary form of the Holtsmark theory.

Consider a neutral impurity atom and ask what is the probability $W(r) d r$ that there is no ionized impurity closer than $r$ and one ionized impurity in the spherical shell volume element $4 \pi r^{2} d r$ at $r$. Let there be $\mathrm{N}_{i}$ ionized impurities per $\mathrm{cm}^{3}$. The probability of having one ionized impurity in the shell between $r$ and $r+d r$ is assumed to be given by

$$
p(r, d r)=\left(4 \pi r^{2} d r\right) N_{i} .
$$

The probability of no ionized impurity closer than $r, P(r)$ is obtained from the relation

$$
P(u+d u)=P(u)\left(1-4 \pi u^{2} d u N_{i}\right) .
$$

Thus

$$
d P / P=-4 \pi u^{2} N_{i} d u
$$


which gives after integration

$$
P(r)=\exp \cdot\left[-(4 \pi / 3) r^{3} N_{i}\right] \text {. }
$$

Thus one has

$$
W(r) d r=P(r) p(r, d r)=4 \pi N_{i} r^{2} d r \exp \cdot\left[-(4 \pi / 3) N_{i} r^{3}\right] .
$$

After introducing the parameter $r_{0}$, defined by

$$
(4 \pi / 3) r_{0}^{3}=1 / N_{i}
$$

one may finally write

$$
W(r) d r=\exp \cdot\left(-r / r_{0}\right)^{3} d\left(r / r_{0}\right)^{3}
$$

Since a given $r$ defines an electric field $F$ at a neural impurity site, and an electric field defines a frequency displacement via the Stark effect, Eq. (3-2) also represents the distribution of these last two quantities. Thus if the field is given by $F=(e / 4 \pi \epsilon) \mathrm{r}^{-2}$, substitution into Eq. (3-2) gives

$$
\begin{aligned}
W(F) d F & =C \exp \cdot\left[-\left(F_{o} / F\right)^{3 / 2}\right] d\left(F_{o} / F\right)^{3 / 2} \\
& =(3 / 2 F)\left(F_{0} / F\right)^{3 / 2} \exp \cdot\left[-\left(F_{o} / F\right)^{3 / 2}\right] d F,
\end{aligned}
$$

where $F_{0}=(e / 4 \pi \epsilon) r_{0}^{-2}$. C was taken as minus one, in order that the field probability be normalized to

$$
\int_{0}^{\infty} W(F) d F=1 \text {. }
$$

For the linear Stark, effect $\Delta \omega=s F$ and $\Delta \omega_{0}=s F_{0}$, where $s$ is a constant. Using a delta function for $I(F, \omega)$, one obtains from Eq. (2-1)

$$
\begin{aligned}
I(\Delta \omega) & =\int_{0}^{\infty} \delta(\Delta \omega-s F) W(F) d F \\
& =\int_{0}^{\infty}|s|^{-1} \delta(F-\Delta \omega / s) W(F) d F \\
& =|s|^{-1} W(\Delta \omega / s) .
\end{aligned}
$$

Substituting for $W(\Delta \omega / s)$ from $E q .(3-3)$, this becomes 


$$
I(\Delta \omega)=(s /|s|)(3 / 2 \Delta \omega)\left(\Delta \omega_{0} / \Delta \omega\right)^{3 / 2} \exp \cdot\left[-\left(\Delta \omega_{0} / \Delta \omega\right)^{3 / 2}\right] .
$$

One obtains two peaks corresponding to positive and negative s, each having the intensity distribution function $I(\Delta \omega)$ as given by Eq. $(3-5)$.

For the quadratic Stark effect $\Delta \omega=t F^{2}$ and $\Delta \omega_{0}=t F_{0}{ }^{2}$, where $t$ is another constant. Again using Eqs. $(2-1)$ and $(3-3)$ this gives

$$
\begin{aligned}
& I(\Delta \omega)= \int_{0}^{\infty} \delta\left(\Delta \omega-t F^{2}\right) W(F) d F \\
&= \int_{0}^{\infty}|t|^{-1} \delta\left(F^{2}-\Delta \omega / t\right) W(F) d F \\
&=(1 / 2|t|)(\Delta \omega / t)^{-1 / 2} \int_{0}^{\infty}\left\{\delta\left[F-(\Delta \omega / t)^{1 / 2}\right]\right. \\
&\left.+\delta\left[F+(\Delta \omega / t)^{1 / 2}\right]\right\} W(F) d F \\
&=(1 / 2|t|)(\Delta \omega / t)^{-1 / 2} W\left[(\Delta \omega / t)^{1 / 2}\right],
\end{aligned}
$$

since $F$ is positive. Or finally, using Eq. $(3-3)$

$$
I(\Delta \omega)=(t /|t|)(3 / 4 \Delta \omega)\left(\Delta \omega_{0} / \Delta \omega\right)^{3 / 4} \exp \cdot\left[-\left(\Delta \omega_{0} / \Delta \omega\right)^{3 / 4}\right] .
$$

For any given energy level $t$ can be either positive or negative, depending on the specific energy, level structure.

The foregoing elementary considerations, which yield the binary approximations to the intensity distribution Eq. $(3-5)$ and $(3-6)$, have been superseded by the work of Holtsmark $(1919,1924)$ and others, who included the cooperative effect of many ions. Review articles on this field where written by Chandrasekhar (1943), Breene Jr. (1957), and Margenau and Lewis (1959).

The field F, which appears in Eq. (3-3), must be written as a vector sum when it is compounded from the fields of many impurities in different places: 


$$
\mathrm{F}=\left|\sum_{\mathrm{n}} \overrightarrow{\mathrm{F}}_{\mathrm{n}}\right|
$$

The analysis leading to Eq. (3-3) must be carried out in a configuration space of $3 \mathrm{~N}$ dimensions. In place of Eq. (3-3) it yields the function $W(\beta) d \beta$ plotted in Figure 12 (curve labelled Holtsmark), where $\beta=F / F_{0}$. For small and large values of $\beta$ one obtains respectively the two alternate expressions:

$$
\begin{aligned}
W(\beta) & =(2 / \pi \beta) \int_{0}^{\infty} v \sin v \exp \cdot\left[-(v / \beta)^{3 / 2}\right] d v \\
& =\left\{\begin{array}{l}
(4 / 3 \pi) \beta^{2}\left(1-0.463 \beta^{2}+0.1227 \beta^{4}+\ldots\right), \beta \text { small } \\
1.496 \beta^{-5 / 2}\left(1+5.107 \beta^{-3 / 2}+14.93 \beta^{-3}+\ldots\right), \beta \text { large. }
\end{array}\right.
\end{aligned}
$$

In the binary theory $F_{0}$ was defined by

$$
\begin{aligned}
\mathrm{F}_{0} & =(e / 4 \pi \epsilon) \mathrm{r}_{0}{ }^{-2}=(\mathrm{e} / 4 \pi \epsilon)\left[(4 \pi / 3) \mathrm{N}_{i}\right]^{2 / 3} \\
& =2.60(\mathrm{e} / 4 \pi \epsilon) \mathrm{N}_{\mathrm{i}}^{2 / 3} .
\end{aligned}
$$

The many-ion calculation gives nearly the same parameter:

$$
F_{0}=2.61(e / 4 \pi \epsilon) \quad N_{1}^{2 / 3} \text {. }
$$

one then passes to the frequency distribution in the same way as before: In the linear Stark effect $\Delta \omega / \Delta \omega_{0}=F / F_{0}=\beta$, and since in general

$$
I(\Delta \omega) d(\Delta \omega)=W(\beta) d \beta,
$$

one finds

$$
I(\Delta \omega)=1 / \Delta \omega_{0} W\left(\Delta \omega / \Delta \omega_{0}\right)
$$

For the quadratic Stark effect $\left(\Delta \omega / \Delta \omega_{0}\right)^{1 / 2}=F / F_{0}=\beta$, and Eq. $(3-3)$ gives

$$
I(\Delta \omega)=1 / 2\left(\Delta \omega_{0} \Delta \omega\right)^{-1 / 2} W\left[\left(\Delta \omega / \Delta \omega_{0}\right)^{1 / 2}\right]
$$


4. The Effect of Screening

When the crystal contains $N_{1}$ ionized impurities per $\mathrm{cm}^{3}$, there will also be an equal number $p=N_{1}$ of free charge carriers, the influence of which has been neglected so far. The effect of these free charge carriers may be approximated by using a screened Coulomb potential of the form

$$
U,=(e / 4 \pi \epsilon) \quad 1 / r e^{-r / \lambda}
$$

Where $\lambda$ is the Debye-Huckel (1923) screening length. In classical statistics

$$
\lambda=\left[\mathrm{kT} / 4 \pi \mathrm{pe} \mathrm{e}^{2}\right]^{1 / 2}
$$

$k$ being the Boltzmann constant.

Differentiating the expression for $U$ in Eq. (4-1) one obtains the screened Coulomb field

$$
\vec{F}=\left(e \vec{r} / 4 \pi \epsilon r^{2}\right)[(1 / r)+(1 / \lambda)] e^{-r / \lambda},
$$

which is to replace the simple Coulomb field in Eq. (3-3). However, analysis of the line-broadening problem even with this simplified field remains formidable. Using the same approach as Ecker (1957), $\vec{F}$ has been approximatéd by

$$
\vec{F}=\left\{\begin{array}{cl}
e \vec{r} / 4 \pi \epsilon r^{3} & \text { if } r<\lambda . \\
0 & \text { if } r \geqslant \lambda .
\end{array}\right.
$$

Figure 10 shows a comparison between the screened field at $90^{\circ} \mathrm{K}$ and the Coulomb field with its cut-off at $r=\lambda$ for three concentrations of impurities, as a function of $r$ in units of $r_{s}$. Where the parameter is defined by

$$
(4 \pi / 3) \quad r_{s}^{3}=1 / N_{A} \text {. }
$$

Using Eq. (4-4), the method of section 3 leads to a dependence of the line shape on the screening parameter 


\section{FIGURE 10.}

Screened electric field (dotted lines), and Coulomb field with cut-off (full lines), as a function of separation. The separation between field point and field producer is expressed in units of the parameter $\left(x_{s}\right)$. The boron concentrations are: i) $1.0 \times 10^{14} \mathrm{~cm}^{-3}$, ii) $1.2 \times 10^{15} \mathrm{~cm}^{-3}$, and iif) $1.2 \times 10^{16} \mathrm{~cm}^{-3}$. The screened electric fields were calculated from the equation $F=(e / 4 \pi \epsilon) r^{-1}\left(x^{-1}+\lambda^{-1}\right) e^{-r / \lambda}$, using the calculated screening length $\lambda$ at $90^{\circ} \mathrm{K}$. 


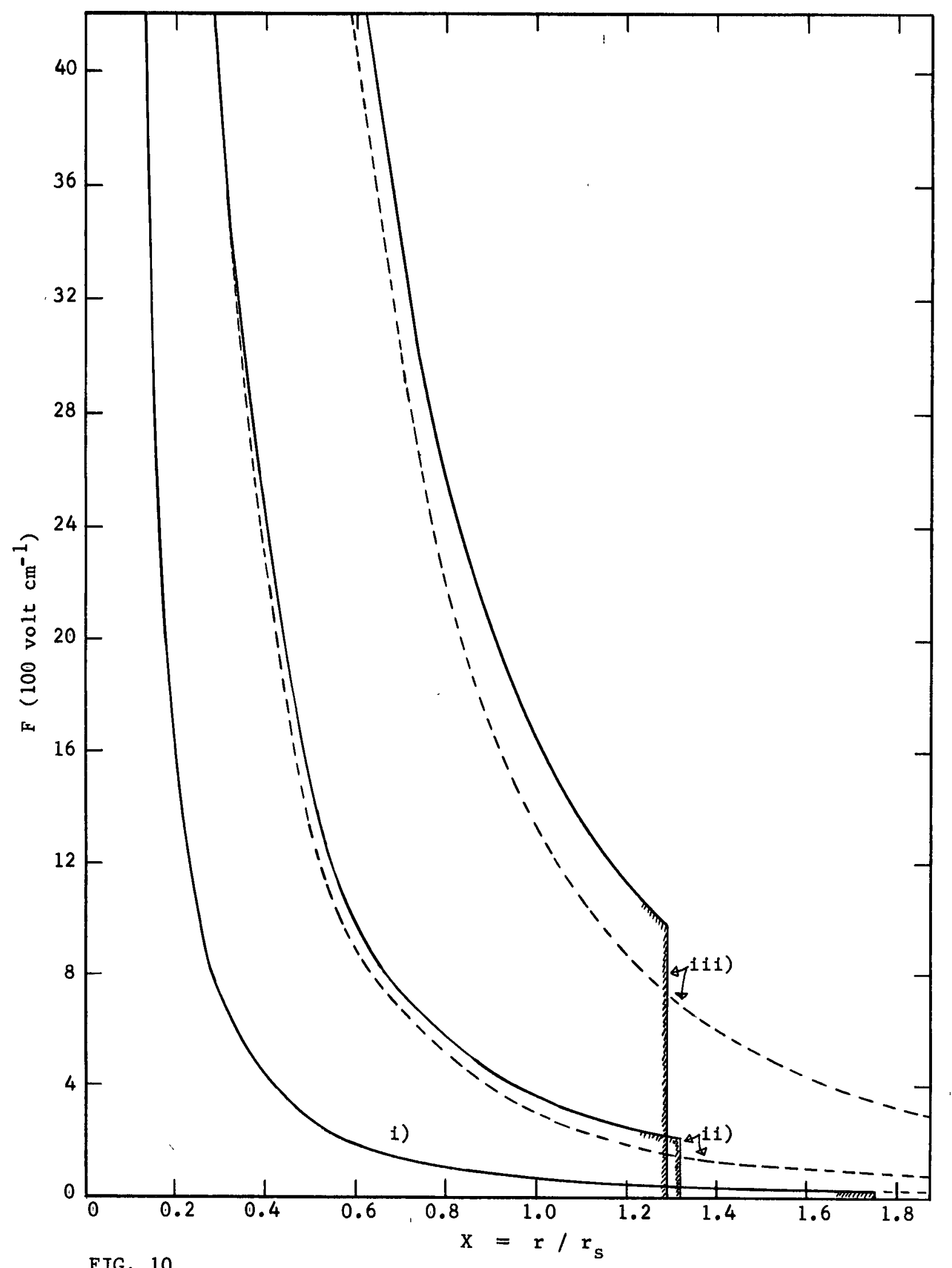




$$
\delta \equiv\left(4 \pi \lambda^{3} / 3\right) N_{i}=(1 / 6) \pi^{-1 / 2}\left(k T / e^{2}\right)^{3 / 2} p^{-1 / 2} .
$$

The physical meaning of the screening parameter is the number of ionized impurities within the Debye radius $\lambda$. Clearly as $\lambda$ tends to infinity the results must agree with those of Holtsmark. Figure 12 shows the results of Ecker's (1957) computations for several values of $\delta$. From these curves one can obtain the half-widths $h(\beta)$, which have been plotted in Figure 13 as functions of the screening parameter. The dependence of the screening parameter on temperature has been shown in Figure 11 for three different concentrations of impurities. Figure 11 was obtained by using Eq. (4-6) and the values for $p$ from Figure 9.

5. Temperature Dependence of the Half-width due to the Linear and the Quadratic Stark Effect

For the linear Stark effect

$$
\Delta \omega=s F=s F_{\circ} \beta,
$$

and hence in units of frequency, the half-width of $I(\Delta \omega)$ is given. by

$$
h_{1}(\Delta \omega)=s F_{0} h(\beta) \text {. }
$$

For the quadratic stark effect

$$
\Delta \omega=t F^{2}=t F_{0}^{2} \beta^{2} \text {, }
$$

and hence in units of frquency, the half-width of $I(\Delta \omega)$ is given by

$$
h_{2}(\Delta \omega)=t F_{0}^{2} h^{2}(\beta) \text {. }
$$

Taking for silicon the dielectric constant $\epsilon / \epsilon_{0}=12$, one finds, using Eq. $(3-8)$

$$
\mathrm{F}_{\mathrm{o}}=2.61(\mathrm{e} / 4 \pi \epsilon) \mathrm{N}_{\mathrm{i}}^{2 / 3}=3.13 \times 10^{-8} \mathrm{~N}^{2 / 3} \text { volt } \mathrm{cm}^{-1} \text {, }
$$

where $\mathrm{N}_{1}$ is in units of $\mathrm{cm}^{-3}$.

Using Eqs. $(5-1),(5-2),(5-3)$, and Figures 11 and 13 , the curves for $h_{1}(\Delta \omega) / s$ and $h_{2}(\Delta \omega) / t$ as a function of temperature for three 
Screening parameter, $\delta$, vs. temperature for boron concentrations of i) $1.0 \times 10^{14} \mathrm{~cm}^{-3}$, ii) $1.2 \times 10^{15} \mathrm{~cm}^{-3}$, and iii) $1.2 \times 10^{16} \mathrm{~cm}^{-3}$. 
Screening parameter, $\delta$

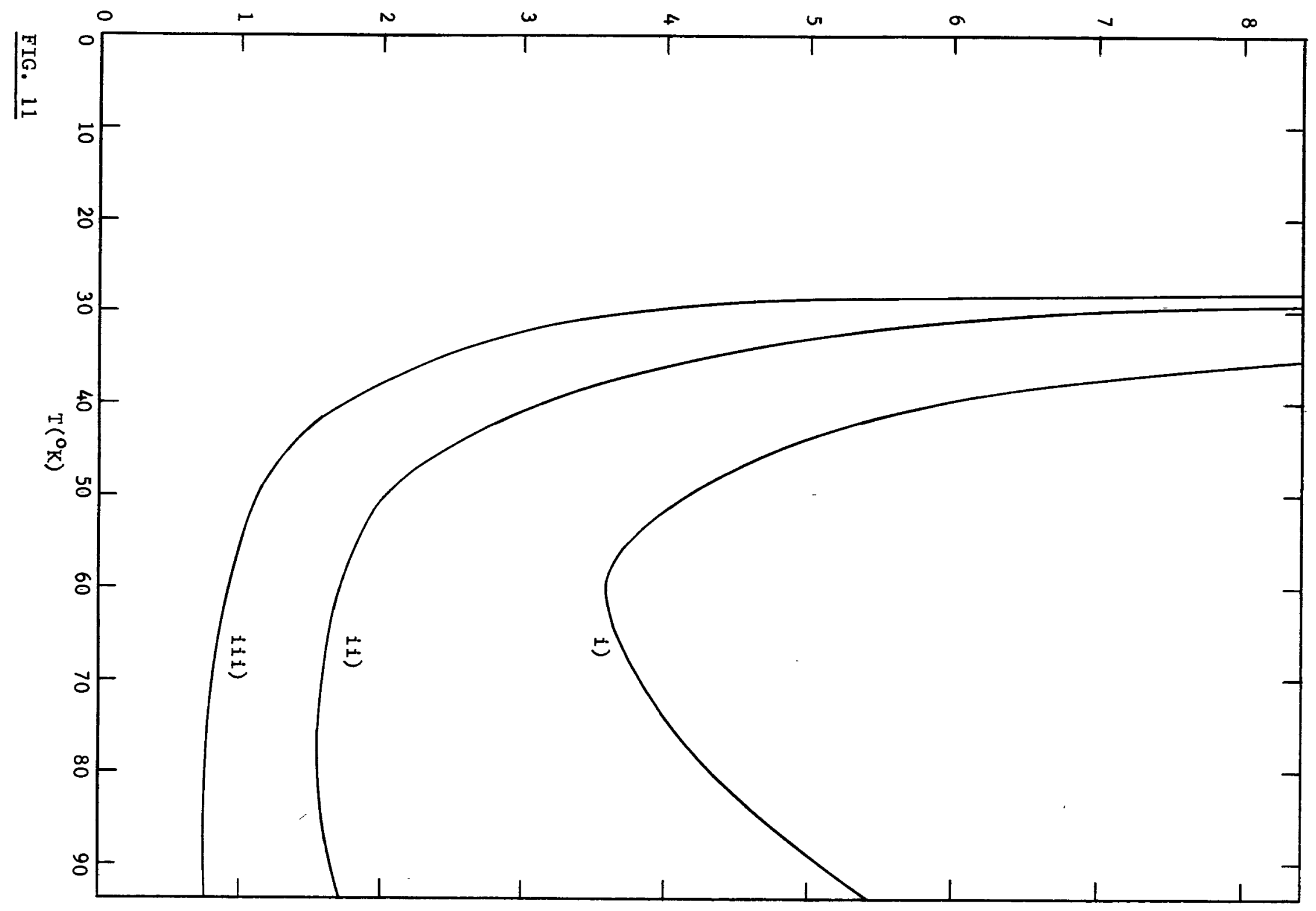




\section{FIGURE 12.}

Field distribution function, $W(\beta)$, for various values of the screening parameter, $\delta$. (Ecker 1957). 


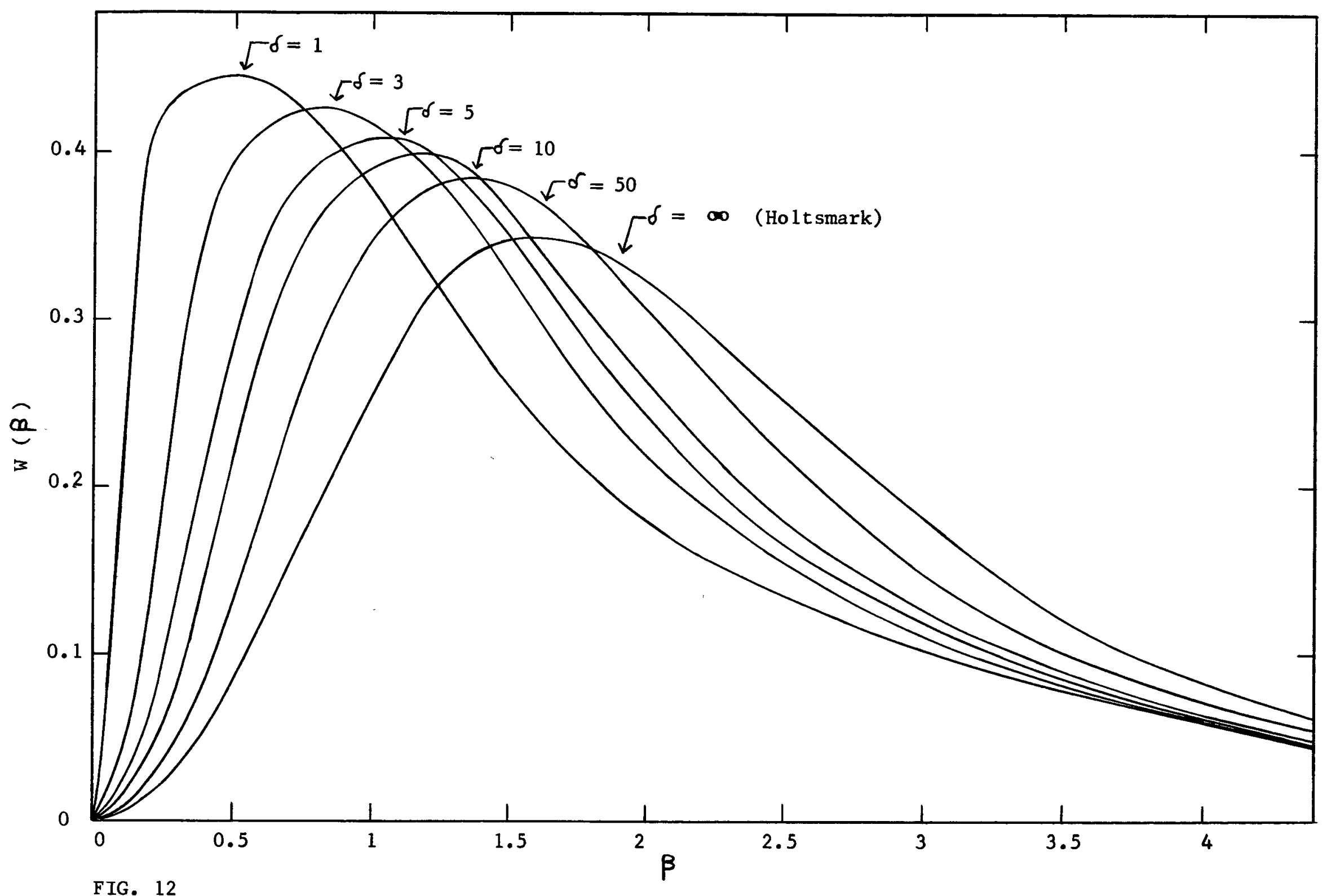




\section{FIGURE $\cdot 13$.}

Half-width of the field distribution, $h(\beta)$, as a function of the screening parameter, $\delta$;

(logarithmic scale). 


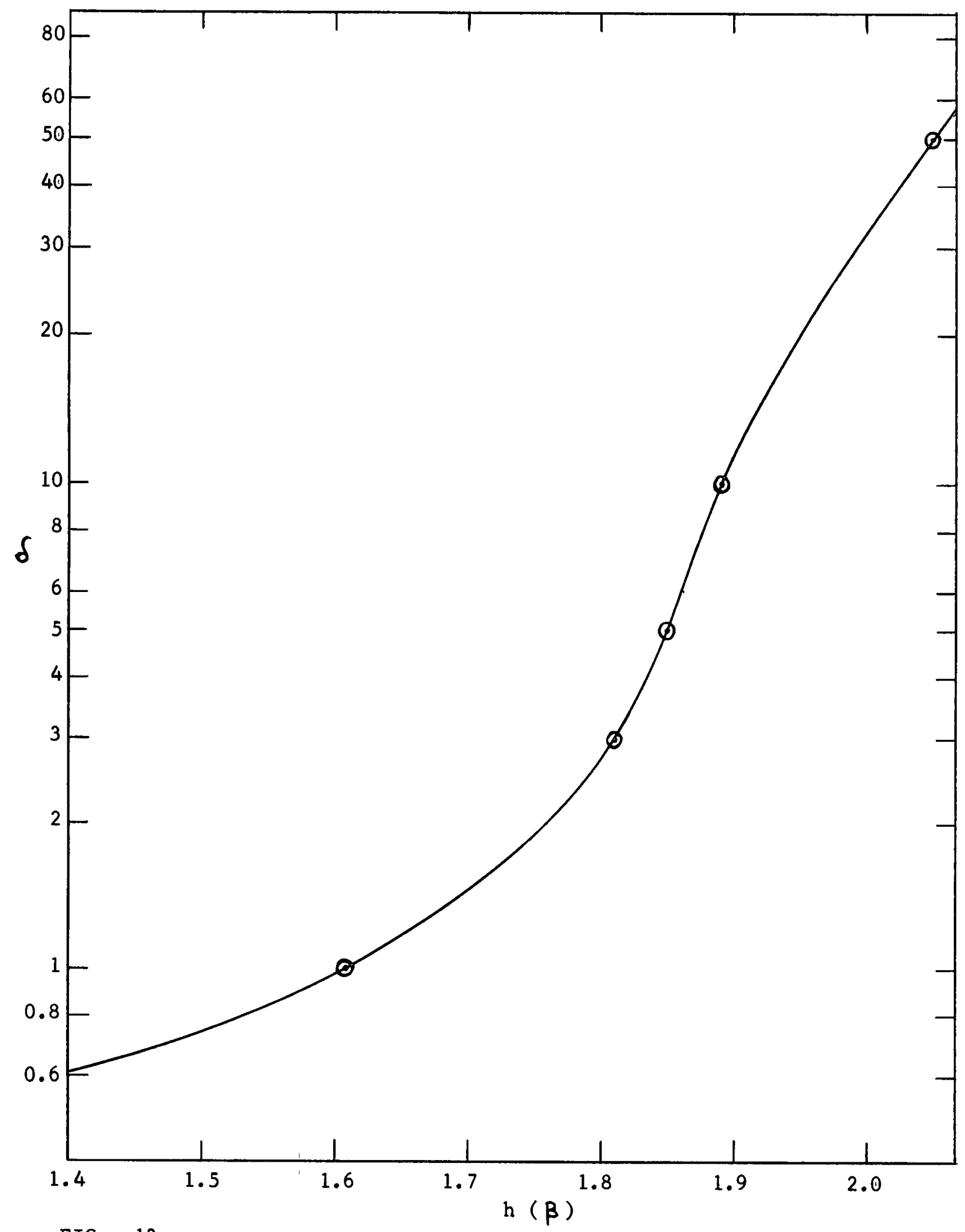

FIG. 13 
impurity concentrations have been calculated. These graphs are shown in Figures 14 and 15 respectively, and their temperature dependence must be compared with Figure 6 , which was obtained by subtracting the half-width extrapolated to zero concentration of impurities (Figure 4) from the true half-width in Figure 5.

Let us assume that the electric field at the impurity center has its direction along the z-axis. The perturbation Hamiltonian is then

$$
\delta V=-F \text { e }
$$

The wave functions of the bound carrier have the form

$$
\psi(i)(\vec{r})=\sum_{j} \alpha_{j} F_{j}(i)(\vec{r}) \phi_{j}(\vec{r})
$$

in the effective mass theory of donor or acceptor states. Here the $\alpha_{j}$ are numerical coefficients, the $\phi_{j}(\vec{r})$ Bloch waves, and the $F_{j}(\vec{i})(\vec{r})$ are hydrogen-like envelope functions. The matrix element of $\delta \mathrm{V}$ between two such states is given by

$$
\left(\psi^{(1)}, \delta V \psi^{\left(1^{\prime}\right)}\right)=\sum_{j}\left(F_{j}^{(i)}, \delta V_{j}^{\left(i^{\prime}\right)}\right) \text {. }
$$

Since the effective mass Hamiltonians for both types of states are invariant under inversion and there are no accidental degeneracies, such as the $2 \mathrm{~s}, 2 \mathrm{p}$ degeneracy in hydrogen, the first-order Stark effect vanishes. However, the full Hamiltonian of the impurity problem has only tetrahedral symetry and is not invariant under inversion. As a result, if the effective mass theory is seriously in error, states belonging to the representation $\mathrm{T}_{1}, \mathrm{~T}_{2}$, and $\Gamma_{8}$ can have an appreciable first-order Stark effect. This might possibly be of significance for the acceptor ground state $\left(\Gamma_{8}\right)$ in silicon (Kohn 1957), but the resulting effect on the half-width is difficult to estimate. By Eq. (3-5) one would presumably obtain two peaks, corresponding to positive and negative strength parameter, whose unresolved resultant may give rise to 


\section{FIGURE 14.}

Half-width in units of the strength parameter s vs. temperature for the linear Stark effect. The boron concentrations are: i) $1.0 \times 10^{14} \mathrm{~cm}^{-3}$,

ii) $1.2 \times 10^{15} \mathrm{~cm}^{-3}$, and iii) $1.2 \times 10^{16} \mathrm{~cm}^{-3}$. 


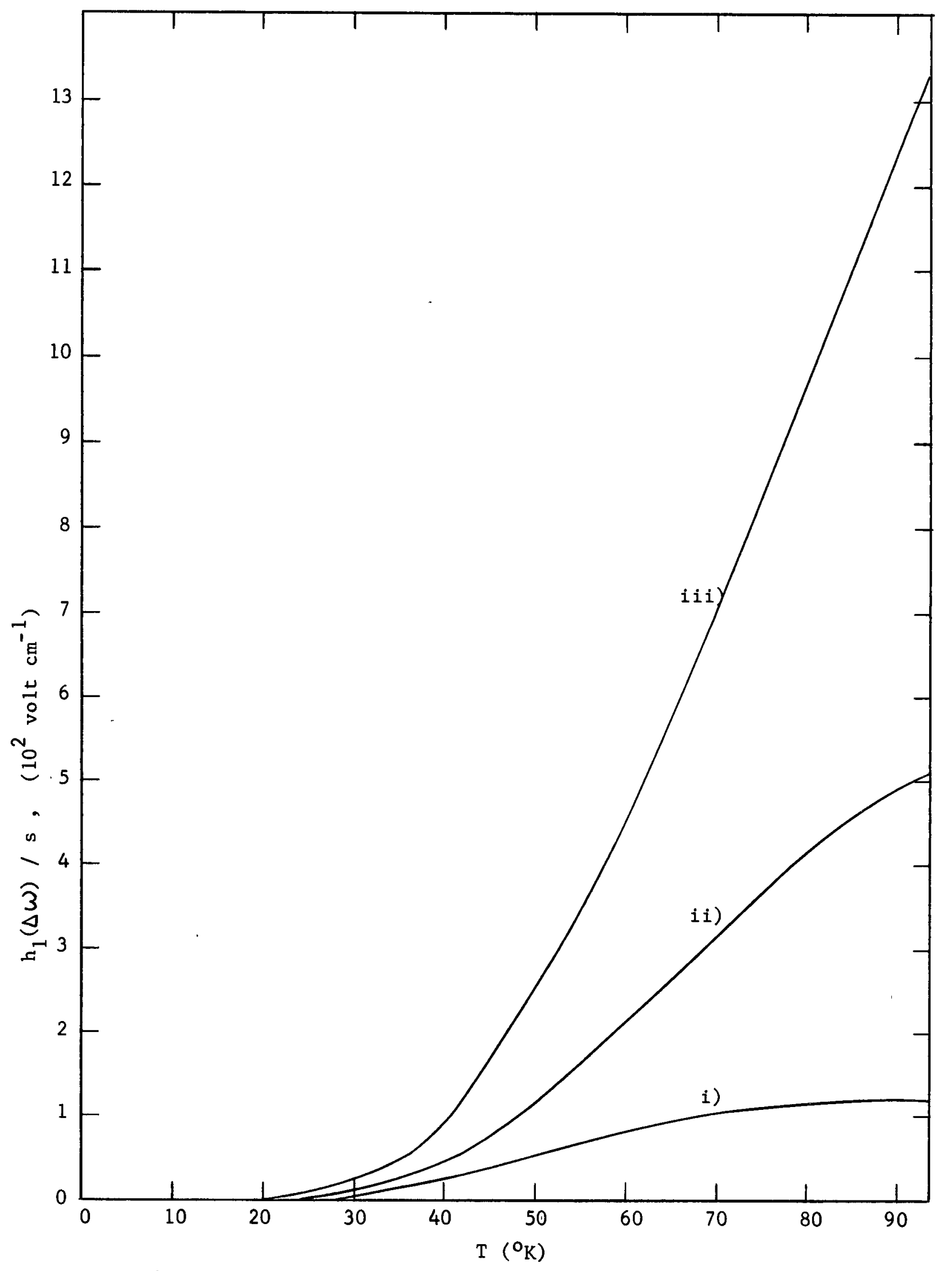

FIG. 14 


\section{FIGURE 15.}

Half-width in units of the strenght parameter $t$ vs.

temperature for the quadratic Stark effect. The

boron concentrations are: i) $1.0 \times 10^{14} \mathrm{~cm}^{-3}$,

11) $1.2 \times 10^{15} \mathrm{~cm}^{-3}$, and 1ii) $1.2 \times 10^{16} \mathrm{~cm}^{-3}$.

To follow page 28. 


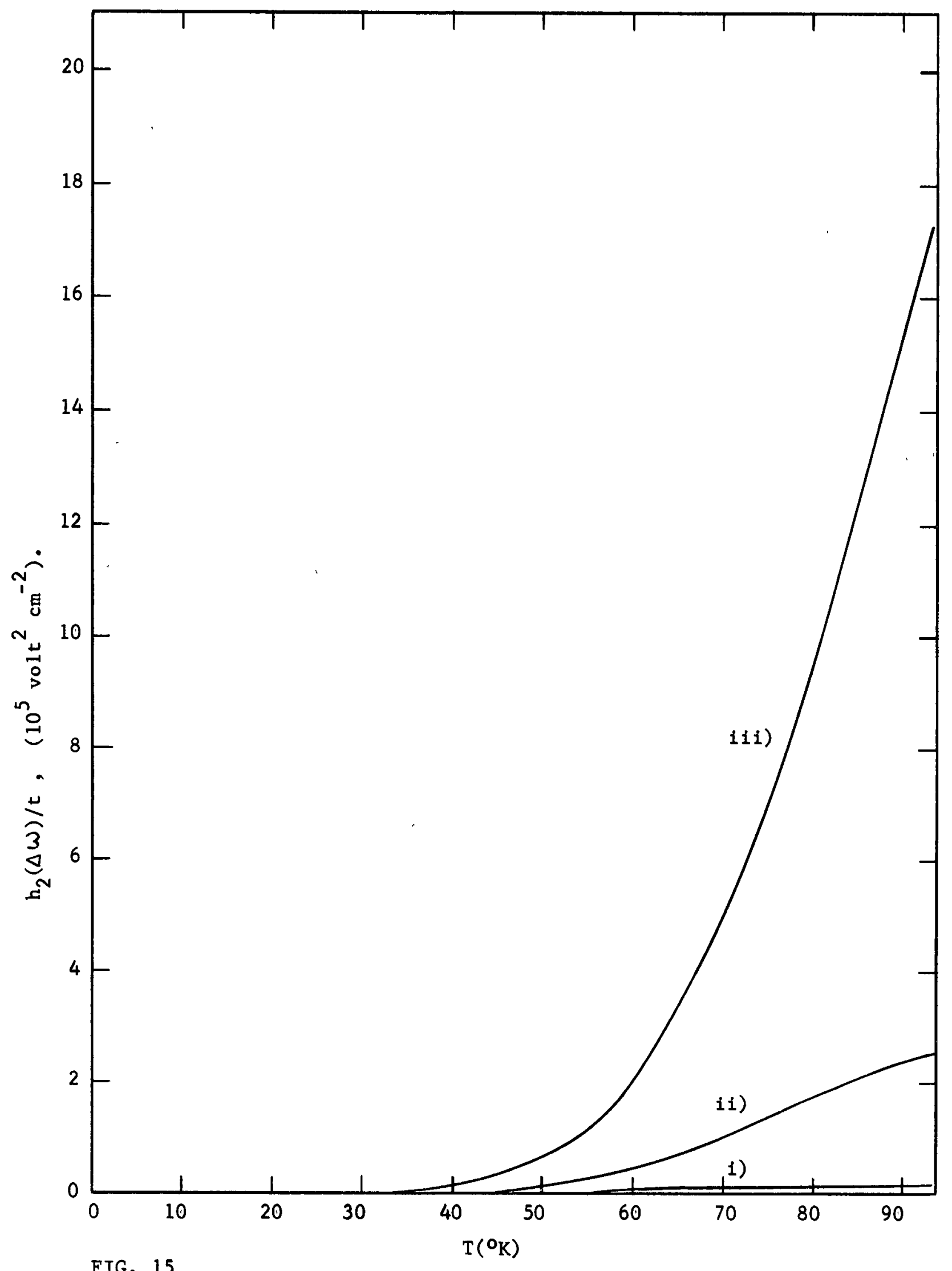

FIG. 15 
an appreciable broadening of the absorption line. Fortunately, however, Figures 6,14 , and 15 seem to suggest by their temperature dependence that the second-order stark effect might be the dominant effect and, as will be seen in the next section, sufficient to explain the experimental data. If the broadening in Figure 6 were caused predominantly, by the first-order Stark effect a steeper rise of the half-width at low temperature would have to be expected, as will be seen from Figure 15, especially for the 1.3 ohm cm material.

6. Evaluation of the Half-width due to the Quadratic Stark Effect

The second-order Stark shift for a nondegenerate state $\psi^{(i)}$ is given by

$$
\left.\delta_{2} E^{(i)}=\sum_{i^{\prime}} \cdot\left|\left(\psi^{(i)}, \delta V \psi^{\left(i^{\prime}\right)}\right)\right|^{2} / E^{(i)}-E^{\left(i^{\prime}\right)}\right) .
$$

Replacing the denominators $E^{(i)}-E^{\left(i^{\prime}\right)}$ by an average value $\Delta E$ and assuming that the linear stark effect $\left(\psi^{(i)}, \delta V \psi^{(i)}\right)$ vanishes, Eq. $(6-1)$ becomes

$$
\begin{aligned}
\delta_{2} E^{(i)} & =1 / \Delta E \sum_{i=}^{\prime}\left|\left(\psi^{(i)}, \delta V \psi^{\left(i^{\prime}\right)}\right)\right|^{2} \\
& =1 / \Delta E \sum_{i \prime}^{\prime}\left(\psi^{(i)}, \delta V \psi^{\left(i^{\prime}\right)}\right)\left(\psi^{\left(i^{\prime}\right)}, \delta V \psi^{(i)}\right) \\
& =1 / \Delta E\left(\psi^{(i)},(\delta V)^{2} \psi^{(i)}\right) \\
& =1 / \Delta E\left(F^{(i)},(\delta V)^{2} F^{(i)}\right) .
\end{aligned}
$$

The shifts will be of a similar order of magnitude for degenerate states. Using Eq. (5-4) for $\delta \mathrm{V}$ and simple hydrogenic wave functions for $\mathrm{F}^{(i)}$, Eq. (6-2) has been evaluated in Appendix II. The result is

$$
\delta_{2} E=t F^{2}=c(e F a *)^{2} / \Delta E \text {. }
$$

Here $a *$ is the effective Bohr radius, and $c$ is a constant which turns out to be 1 for the 1 s state and 18 . for the $2 \mathrm{p}_{0}$ state (Appendix II). Thus, the 
quadratic Stark effect may be neglected for the ground state, when compared with that for the excited state.

What is the value of $t$ required, such that $h_{2}(\Delta \omega) / t$ in Figure 15 gives the temperature dependence of the half-width in Figure 6 ? The curve for the $1.3 \mathrm{ohm} \mathrm{cm}$ boron-doped silicon seems best suited for numerical comparison, since it shows the largest change with temperature.

From Figure 6 the change in half-width between $0^{\circ} \mathrm{K}$ and $80^{\circ} \mathrm{K}$ is

$$
\Delta\left(h_{c}^{\prime \prime}-h_{o}^{\prime \prime}\right)=1.06 \mathrm{mev} .
$$

Over the same temperature range one obtains from Figure 15:

$$
\Delta\left[h_{2}(\Delta \omega) / t\right]=0.95 \times 10^{6} \text { volt } \mathrm{cm}^{-2} \text {. }
$$

Equating $\Delta\left[h_{2}(\Delta \omega)\right]$ and $\Delta\left(h_{c}^{\prime \prime}-h_{0}^{\prime \prime}\right)$ one obtains

$$
t=1.1 \times 10^{-6} \mathrm{mev} \text { volt } \mathrm{t}^{-2} \mathrm{~cm}^{2} \text {. }
$$

Let us assume that energy level 4 (Figure 8) may be approximated by a hydrogenic $2 p$ state. The experimental value for its binding energy is

$$
E_{2}=(46-39.7) \mathrm{mev}=6.3 \mathrm{mev} \text {. }
$$

Using this value and the hydrogenic energy equation

$$
E_{n}=-m^{*} e^{4} / 2 x^{2} k^{2} n^{2}=-e^{2} / 2 K a * n^{2},
$$

with $n=2$, one obtains for the effective Bohr radius of state 4:

$$
a *=24 \AA \text {. }
$$

Using Eqs. (6-6), (6-9), and taking $c=18$ for a 2 po state, Eq. (6-3) leads to

$$
\Delta E=c e^{2} a *^{2} / \mathrm{t}=1 \mathrm{mev}
$$

which has the order of magnitude one would expect from the general nature of the energy, level diagram (Figure 4).

In equating $\Delta\left[h_{2}(\Delta \omega)\right]$ and $\Delta\left(h_{c}^{\prime \prime}-h_{0}^{\prime \prime}\right)$ it was assumed implicitly, that the interaction between neutral impurities, which is responsible for the low temperature broadening, changes little with temperature below $80^{\circ} \mathrm{K}$. 
Screening and a decrease of $N_{A}-N_{1}$ will tend to reduce this contribution to the half-width with increasing temperature. However, since these forces will be appreciable only for nearest neighbors, for which the screening is small below $80^{\circ} \mathrm{K}$, the temperature dependence of their contribution to the half-width should be relatively small below $80^{\circ} \mathrm{K}$.

\section{Statistical Broadening due to van der Waals Forces}

In this and the following section the interaction between neutral impurities will be discussed. The present section, deals with van der Waals forces, which arise when the overlap between the wave functions is negligible. All other forces between neutral atoms may be classed as "overlap forces" and arise only when the wave function of one atom overlaps that of the other. These forces will be considered in the following section. Margenau (1933). developed the statistical theory for broadening by van der Waals forces in order to explain pressure broadening in gases. The line shape of this distribution is

$$
I(\Delta \nu)=2 \pi b^{1 / 2} N \Delta \nu^{-3 / 2} \exp \cdot\left(-4 \pi^{3} N^{2} / 9 \Delta \nu\right) \text {. }
$$

The half-width of this distribution is

$$
h_{w}(\Delta \nu)=0.82 \pi^{3} b N^{2} \text {. }
$$

Where $b$ is the strength constant of the van der Wals forces. That is

$$
v_{i}=-b r_{1}^{-6}
$$

is the van der Waals interaction between the absorbing impurity and the ith neutral impurity, and $r_{1}$ is the distance between them.

When applied to the present problem $N=N_{A}-N_{i}$ is the number of neutral impurities per $\mathrm{cm}^{3}$. Thus at very low temperatures one has $N=N_{A}$. Figure 6 shows that at low temperatures when the number of ionized impurities is negligible, the half-widths are $0.03,0.12$, and $0.60 \mathrm{mev}$, for 
impurity concentrations of $(1,12$, and 120$) \times 10^{14} \mathrm{~cm}^{-3}$ respectively. If the van der Waals forces were to explain this concentration broadening at low temperatures, then according to Eq. (7-2) the half-width should increase with the square of the concentration. That is, they should be in the ratios

$1: 144: 14400$. However, they are observed to go like $1:: 4: 20$.

If the expression by London and Eisenschitz (1930) for the strength parameter $b$ is modified to suit the present problem, one obtains

$$
b=6.48\left(e^{2} / K a *\right) a * 6 \text {. }
$$

Substituting this 1nto Eq. (7-2), and taking $K=12$ for silicon, leads to

$$
h_{w}(\Delta \nu)=2.0 \times 10^{-3} a * 5 \mathrm{~N}^{2} \mathrm{mev},
$$

where $a^{*}$ is in $\mathrm{cm}$ and $\mathrm{N}$ in $\mathrm{cm}^{-3}$. Taking for the effective Bohr radius of the ground state a value of $a *=13 \AA$, Eq. $(7-5)$ gives for $N=1.2 x$ $10^{16} \mathrm{~cm}^{-3}$

$$
h_{w}(\Delta v)=1.1 \times 10^{-5} \text { mev. }
$$

This has to be compared with an experimental half-width $\left(h_{c}^{\prime \prime}-h_{0}^{\prime \prime}\right)$ of $0.60 \mathrm{mev}$. Thus it would seem that the van der Waals interaction is too weak to explain the varlation of the half-width with concentration of impurities at low temperatures (Figure 6).

\section{Broadening due to Overlap Forces}

In this section it will be shown in a qualitative way how overlap forces may account for the observed early onset of concentration broadening at low temperatures (F1gure 6).

Baltensperger's (1953) work, the relevant part of which is shown in Figure 16, suggests that concentration broadening should start at

$$
r_{s}=12 a^{*} \text {. }
$$

Here a* is the effective Bohr radius of a bound carrier of effective mass $\mathrm{m}^{*}$ 


\section{FIGURE 16.}

Broadening of hydrogenic levels vs. distance between

impurities in units of the effective Bohr radius, a*. (Baltensperger 1953).

To follow page 32 . 


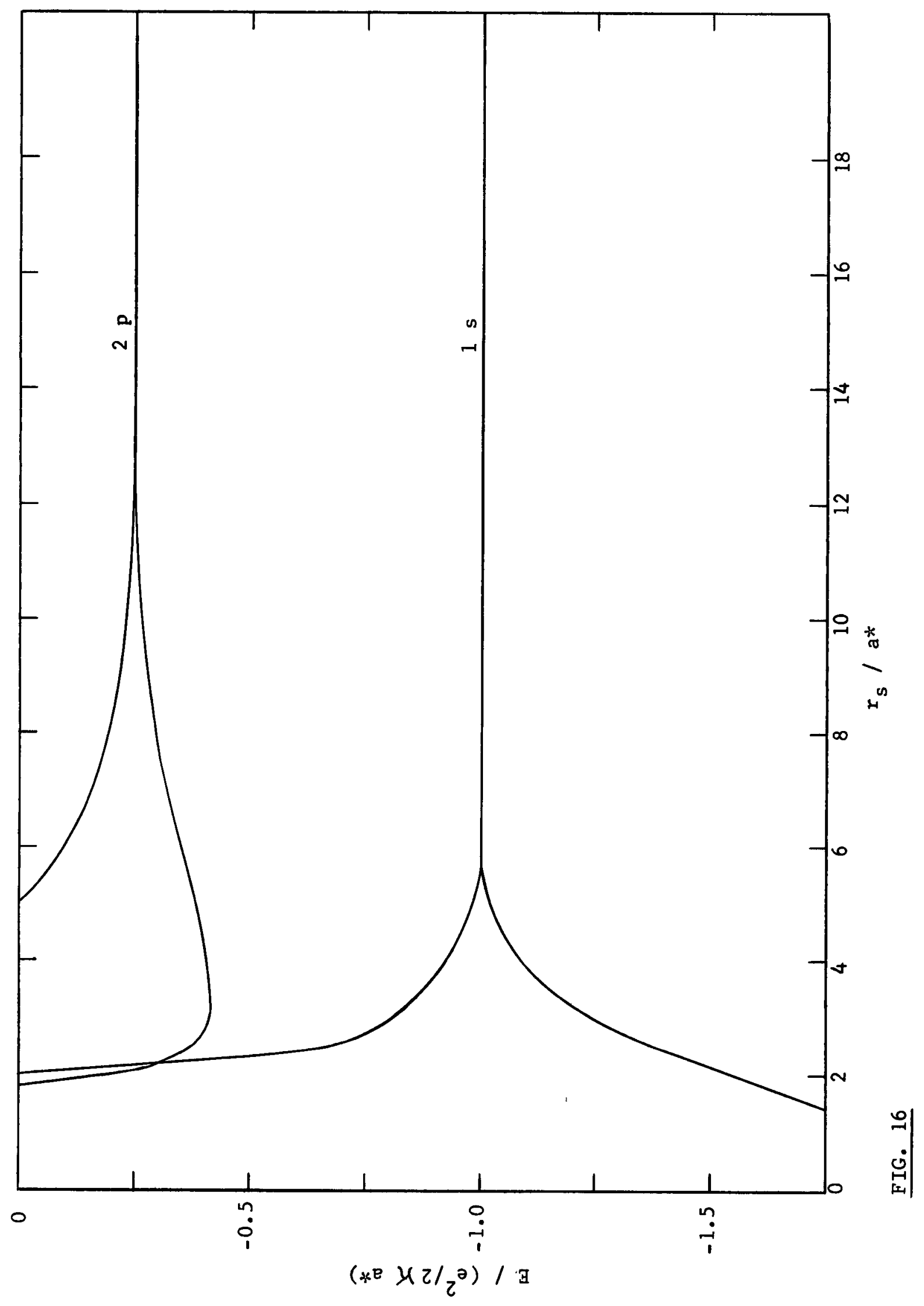


in a crystal with dielectric constant $\mathcal{K}$, and $r_{s}$ is defined by

$$
(4 \pi / 3) \mathrm{r}_{\mathrm{s}}^{3}=1 / \mathrm{N}_{\mathrm{A}} \text {. }
$$

If one takes (Conwell 1956) for boron-doped silicon $K=12, \mathrm{~m}^{*} / \mathrm{m}=0.5$, and hence

$$
a^{*}=K a m / m^{*}=13 \AA,
$$

then Eqs. $(8-1)$ and $(8-2)$ would suggest that concentration broadening should set in at about

$$
\mathrm{N}_{\mathrm{A}}=6 \times 10^{16} \mathrm{~cm}^{-3} \text {. }
$$

This, as was mentioned earlier, seemed to agree with Newman's (1956) data. In contrast to this, however, the present data (Figure 6) shows that concentration broadening starts below

$$
\mathrm{N}_{\mathrm{A}}=1.2 \times 10^{15} \mathrm{~cm}^{-3} \text {. }
$$

How can one bring Baltensperger's calculated values in agreement with this earlier onset of broadening? Two ways suggest themselves. The first involves replacing $r_{S}$ in $E q .(8-1)$ by $X r_{S}$, where $X<1$. This takes care of the fact that the impurities do not form a regular lattice as assumed by Baltensperger (1953). The second modifiction, that of $a^{*}$, takes into consideration that the simple hydrogenic model used to obtain Figure 16 gives rather inadequate values for the binding energy. Instead of a regular spacing of impurities, let us assume a random distribution. Since for overlap forces we are only concerned with small separations, we may use the binary approximation of Holtsmark's theory. By Eq. (3-2) the probability of a nearest neighbor at a distance $\mathrm{r}=\mathrm{Xr}_{\mathrm{s}}$ is given by

$$
W(x) d x=\exp \cdot\left(-x^{3}\right) \cdot d x^{3} \cdot
$$

Now let us ask the question: "What is the value of $\mathrm{x}=\mathrm{X}_{1 / 2}$, such that half the impurities find themselves closer than $x_{1 / 2} r_{s}$ to the neighboring one, 
and half of them are further away?" Eq. $(8,6)$ gives

$$
1 / 2=\int_{0}^{x_{1} / 2} \exp \cdot\left(-x^{3}\right) d x^{3}=1-\exp \cdot\left(-x_{1 / 2}^{3}\right),
$$

or,

$$
x_{1 / 2}=0.885
$$

The mean spacing of the impurities closer than $x_{1 / 2} r_{s}$ to their nearest neighbor is $0.648 \mathrm{r}_{\mathrm{S}}$, which suggests that the effective mean spacing in Eq. (8-1) should be about

$$
\mathbf{r}_{\mathrm{s}}^{*}=0.7 \mathrm{r}_{\mathrm{s}}
$$

For computing the edges of the 1 s and the $2 \mathrm{p}$. bands (Figure 16) Baltensperger (1953) used a simple hydrogenic model in conjunction with the cellular method. This involves assuming the validity of the effective mass Schrödinger equation

$$
\hbar^{2} / 2 \mathrm{~m}^{*} \nabla^{2} \psi+\left(\mathrm{e}^{2} / K_{\mathrm{r}}+\mathrm{E}_{\mathrm{n}}\right) \psi=0
$$

within a sphere of radius $r_{s}$, defined by Eq. (8-2). The general solution of Eq. (8-8) has the form

$$
\psi_{\mathrm{n}, 1, \mathrm{~m}}=\mathrm{R}_{\mathrm{n}, 1}(\mathrm{r}) \mathrm{Y}_{1, \mathrm{~m}}(\theta, \phi),
$$

and is well known from the hydrogen problem.

The energy is given by

$$
E_{n}=-m^{*} e^{4} / 2 K^{2} k^{2} n^{2}=-e^{2} / 2 K a^{*} n^{2} \text {. }
$$

where, in the cellular method, $\mathrm{n}$ is to be determined by appropriate boundary conditions. A value of $a^{*}=13 \AA$ for boron doped silicon gives one the observed Ionization energy $E_{1}=46 \mathrm{mev}$ for the bound hole.

In estimating the concentration where the overlap forces become appreciable, one is concerned with the $2 p$ states, since these broaden long before the 1s state (Figure 16). It is now realized that acceptor states are much more complicated than simple hydrogenic wave functions. However, if one wants to approximate the particular line under study by a simple 
hydrogenic $1 s \rightarrow 2 p$ transition, the most reasonable approach might be to require that $a^{*}$, in the $2 p$ wave function $\psi_{210}$, Eq. (8-10), satisfy Eq. (8-11) with $n=2$. Thus, using the experimental value for the binding energy of $E_{2}=6.3 \mathrm{mev}$ for energy level 4 (Figure 8), one obtains from. Eq. (8-11)

$$
a *=24 \AA \text {. }
$$

With the modifications given by Eqs. (8-7) and (8-12), one obtains from Eqs. $(8-1)$ and $(8-2)$, for the onset of concentration broadening at low temperatures, a value of

$$
\mathrm{N}_{\mathrm{A}}=3 \times 10^{15} \mathrm{~cm}^{-3} \text {, }
$$

which compares rather well with Eq. (8-5), considering the crudeness of the assumptions involved. Not on $1 y$ is the hydrogenic model a rather crude approximation, but the usefulness of the band scheme itself becomes doubtful, when one is dealing with a random distribution of impurities.

\section{Broadening due to Internal Strains}

In this and the following section an attempt is made to account for the half-width, extrapolated to zero impurity concentration (Figure 5). It is believed that an appreciable part of this width results from internal strains due to dislocations. This contribution to the half-width is expected to be essentially temperature independent over the temperature range investigated. The remaining contribution to the half-width will be attributed to phonon broadening in the following section.

The samples used in this experiment have a quoted (Merck and Co. 1962) dislocation density of about $n=5 \times 10^{4}$ dislocation lines per $\mathrm{cm}^{2}$. These dislocation lines are expected to give rise to internal strains whose magnitude is given approximately by (Kohn 1957)

$$
\mathrm{s}=\left(\mathrm{n}^{1 / 2}\right) \times 10^{-8} \mathrm{~cm} \approx 2.2 \times 10^{-6} \text {. }
$$


As discussed in section III- 1 , in the absence of strains all acceptor levels are according to theory either twofold or fourfold degenerate. Under a shear strain s, the fourfold degenerate levels will split into two twofold degenerate levels. . The magnitude of the splitting is

$$
\Delta E=s \in \approx\left(10^{-8} \mathrm{~cm}\right) \mathrm{n}^{1 / 2} \in \approx 0.03 \mathrm{mev} \text {. }
$$

In this and the following section $\epsilon$ is an effective deformation potential constant, which depends on the geometry of the strain and is of the order of $15 \mathrm{ev}$ ( $\operatorname{Lax}$ and Burstein 1955). Thus for $\mathrm{kT}>\mathrm{s} \in$ (i.e. $\mathrm{T}>1 / 2^{\circ} \mathrm{K}$ ), transitions from the ground state to a twofold degenerate level will be broadened by about $0.03 \mathrm{mev}$ due to internal strains; while transitions from the ground state to a fourfold degenerate level will be broadened by about $0.06 \mathrm{mev}$. For $\mathrm{T}<1 / 2^{\circ} \mathrm{K}$ only the twofold degenerate lower energy level will be occupied. Hence no broadening of absorption lines due to the ground state splitting would be expected, and only a transition to a fourfold excited state would be broadened by about 0.03 mev .

\section{Phonon Broadening}

Let us consider absorption line 4, corresponding to the transition from the ground state to the energy level $T_{\beta}$ (level 4 in Figure 8 ). The electron-phonon interaction will cause both this level, and the ground state to have a finite life-time, resulting in a broadening of these levels, and thus also in a broadening of the corresponding absorption line. The magnitude of this broadening will depend on the exact probabilities for the different modes of decay and excitation available to these states. The life-time of the ground state will be much longer than that of the excited state, and hence we shall neglect its contribution to the half-width of the 
absorption line. Let us denote by $\lambda$ the state that has the most influence on the life-time of the state $\beta$. According to Nishikawa (1962), the contribution of this state $\lambda$ to the half-width of the zero-phonon line is

$$
\left.\Delta \omega_{\lambda}=\left(\epsilon^{2} / v^{2} a^{*}{ }^{3} \rho 2 \pi\right) y_{\beta \lambda}^{\prime} \overline{\left[\left|\theta_{\beta \lambda}(\vec{q})\right|^{2}\right.}\right]_{q=y / a^{*}} \times \begin{cases}1+\nu ; & T_{B}>T_{\lambda} \\ \nu ; & T_{\beta}<T_{\lambda},\end{cases}
$$

where

and

$$
\begin{aligned}
y_{\beta \lambda} & =\left|T_{\beta}-T_{\lambda}\right| a^{*} / \hbar v, \\
\nu & =\left(e^{T_{c} / T}-1\right)^{-1},
\end{aligned}
$$

is the characteristic temperature, above. which the half-width starts increasing.

$$
\theta_{\beta \lambda}(\vec{q})=\int \overrightarrow{d r} F_{\beta}(\vec{r})^{*} F_{\lambda}(\vec{r}) \quad e^{i \vec{q} \cdot \vec{r}},
$$

$F(\vec{r})$ being the eigenfunctions of the unperturbed electronic Hamiltonian, which will be approximated by simple hydrogenic functions.

The other parameters have the following meaning:

$$
\begin{aligned}
& \vec{q}=\text { wave number vector of the phonon, } \\
& v=\text { sound velocity in silicon: }=8.3 \times 10^{5} \mathrm{~cm} \mathrm{sec} .^{-1}, \\
& \epsilon=\text { deformation potential constant } \approx 15 \mathrm{ev}, \\
& \rho=\text { density of silicon }=2.33 \mathrm{~g} \mathrm{~cm}^{-3}, \\
& \mathrm{k}=\text { Boltzmann's constant }=1.38 \times 10^{-16} \mathrm{erg} \mathrm{deg}-1
\end{aligned}
$$

Unfortunately, the nature of the states $\beta$, and $\lambda$, the value of ${ }^{T} \lambda$, and the proper choice for the effective Bohr radius a*, are rather uncertain. Let us start with the experimental value $\mathrm{T}_{C} \approx 47^{\circ} \mathrm{K}$ (Figure 5) and the assumption that $\beta$ has $2 \mathrm{p}$ character (Kohn 1957). If one takes $\mathrm{a}^{*}=13.5 \AA$ (Nishikawa 1962), Eq. (10-4) gives $\mathrm{y}_{\beta \lambda}=1$, and thus by Eq. (10-2) one obtains $\left|T_{\lambda}-T_{\beta}\right|=4.0 \mathrm{mev}$. This would suggest identifying the state $\lambda$ 
with the state 2 in Figure 8 . Assuming this state is also 2p-like, one obtains by using simple hydrogenic wave functions

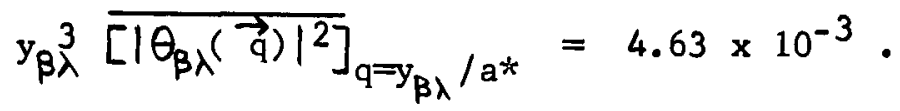

With this value Eq. $(10-1)$ gives

$$
\Delta \omega_{\lambda}=\left\{\begin{array}{l}
0.106 \mathrm{mev} \text { for } \mathrm{T}=\mathrm{T}_{\mathrm{c}} \\
0.170 \mathrm{mev} \text { for } \mathrm{T}=2 \mathrm{~T}_{\mathrm{c}} .
\end{array}\right.
$$

If on the other hand one takes $a^{*}=24 \AA$ (the value one obtains from Eq. (8-11) for the energy $T_{\beta}$, with $\left.n=2\right)$, Eq. (10-4) gives $\mathrm{y}_{\beta \lambda}=1.8$. By Eq. (10-2) this implies $\left|\mathrm{T}_{\beta}-\mathrm{T}_{\lambda}\right|=4.1 \mathrm{mev}$, and thus suggests again identifying the state $\lambda$ with the state 2 in Figure- 8 . However, for $y_{\beta \lambda}=1.8$ one obtains

$$
\mathrm{y}_{\beta \lambda}^{3}\left[\left|\theta_{\beta \lambda}(\vec{q})\right|^{2}\right]_{\mathrm{q}=\mathrm{y}_{\beta \lambda} / \mathrm{a}}=0.70 \times 10^{-3} \text {, }
$$

which after substitution into Eq. (10-1) leads to

$$
\Delta \omega_{\lambda}=\left\{\begin{array}{l}
0.0029 \mathrm{mev} \text { for } \mathrm{T}=\mathrm{T}_{\mathrm{C}} \\
0.0046 \mathrm{mev} \text { for } \mathrm{T}=2 \mathrm{~T}_{\mathrm{C}} .
\end{array}\right.
$$

Another possibility is that the state $\lambda$, responsible for the shortened life-time of $\beta$, is not the $2 p-1$ ike state 2 in Figure 8 , but rather a $2 \mathrm{~s}-1$ ike state, which one may expect in this general region, and which one would presumably not see in optical absorption from the lsolike ground state. Making this assumption one obtains for $a^{*}=13.5 \AA$, corresponding to $y_{\beta \lambda}=1$, that $\Delta \omega_{\lambda}=0$. For $a^{*}=24 \AA$, corresponding to $\mathrm{y}_{\beta \lambda}=1.8$ one obtains

$$
\mathrm{y}_{\beta \lambda}^{3}\left[\left|\theta_{\beta \lambda}(\vec{q})\right|^{2}\right]_{q=y_{\beta \lambda} / a^{*}}=2.75 \times 10^{-3} \text {. }
$$

Substituting this value into Eq. (10-1) one obtains

$$
\Delta \omega_{\lambda}=\left\{\begin{array}{ll}
0.0074 \mathrm{mev} & \text { for } \mathrm{T}=\mathrm{T}_{\mathrm{C}} \\
0.0117 \mathrm{mev} \text { for } \mathrm{T}=2 \mathrm{~T}_{\mathrm{c}}
\end{array} .\right.
$$


If one considers the crudeness of the wave functions used, and the nature of the approximations involved, the agreement between the above estimates, especially, Eq. (10-6), and the width extrapolated to zero concentration in Figure 5, seems to be amazingly good. One may observe, that the increase in width of the extrapolated line in Figure 5 is some. what steeper than that predicted by Eq. (10-6) between $T=T_{C}$ and $\mathrm{T}=2 \mathrm{~T}_{\mathrm{C}}$. However, this would be expected, if one remembers that while the multi-phonon processes are of little importance to the half-width below $T=T_{C}$, their relative importance when compared with the zero-phonon process increases with temperature. They may be expected to make an appreciable contribution to the experimentally observed half-width above. $\mathrm{T}=\mathrm{T}_{\mathrm{c}}$ 
CHAPTER IV

Conclusions

The data presented in this thesis concerning a study of absorption line width in boron-doped silicon, differs considerably from that obtained by previous authors. The reason for this disagreement is a previous lack of sufficient resolution and a fallure to make proper allowance for line distortion by the finite spectrometer slit width. The low temperature half-width is considerably smaller, and its temperature dependence above $50^{\circ} \mathrm{K}$ much steeper than found previously. The onset of concentration broadening is seen to occur at a considerably smaller impurity concentration than seemed to follow from previous experiments. The positions of the absorption lines are essentially in agreement with those obtained by Hrostowski and Kaiser (1958).

It is believed that four effects make significant contributions to the true halfowidths. These are a) statistical stark broadening, b) phonon broadening, c) broadening due to the overlap of impurity wave functions, and d) broadening by internal strains. To the approximation that the absorption lines (corrected for spectrometer distortion) have lorentzian profiles, their half-widths are fust the sums of the widths for the four broadening mechanisms when each is considered by itself. This follows, since the absorption lines may be thought of as resulting from a combination of the separate broadening effects by three consecutive convolution integrals.

In a semiconductor containing a random distribution of neutral and ionized impurities, different absorbing impurities will be in different electric fields due to the surrounding lonized impurities. These fields (F) should be expected to give rise to appreciable second-order Stark shifts 
$\left(\delta_{2} E=t F^{2}\right)$ of the excited states, resulting in a broadening of the total absorption lines. The contribution to the half-width from this effect was obtained from a knowledge of the field strength probability function W(F), which had been extensively studied previously in connection with gravitational problems, gases, and plasmas. Ecker (1957) computed W(F), including the effect of screening by mobile charge carriers. Using these results, and the calculated dependence of the fonized impurity concentration on the temperature, this statistical Stark broadening was found to account satisfactorily for the rapid increase of the half-width above $50^{\circ} \mathrm{K}$. Agreement with the data was achieved by using a strength parameter of $t=1.1 \times 10^{-9}$ ev volts $\mathrm{cm}^{-2}$, which has the magnitude one would expect from an approximate calculation.

At very low impurity concentrations an essential contribution to the half-width is expected to result from the finite life-time of the excited state due to the electron-phonon interactions. Theoretical calculations show that the half-width for this process is given by

$$
\mathrm{h}=\mathrm{h}_{\mathrm{o}} /\left(1-\mathrm{e}^{-\mathrm{T}_{\mathrm{c}} / \mathrm{T}}\right) \text {. }
$$

The calculated value of $h_{0}$ depends on the choice of the characteristic temperature $\mathrm{T}_{c}$. The best agreement between this theory and the data was obtained for $\mathrm{T}_{\mathrm{c}}=47^{\circ} \mathrm{K}$ and $\mathrm{h}_{\mathrm{o}}=1.0 \times 10^{-4} \mathrm{ev}$. For these values the theory suggests that the life-time of the state responsible for the absorption line under study is mostly influenced by a state about $4.0 \times 10^{-3}$ ev below it.

The multi-phonon processes are of little importance to the half-width below $\mathrm{T}=\mathrm{T}_{\mathrm{C}}$, but their relative importance when compared to the zero-phonon processes increases with temperature. They may be expected to make an appreciable contribution to the experimentally 
observed half-width above $\mathrm{T}=\mathrm{T}_{\mathrm{C}}$.

In addition, a nearly temperature independent contribution to the half-width of about $3 \cdot x \cdot 10^{-5}$ ev can be expected from internal strains due to dislocations (corresponding to a quoted dislocation density of about $5 \times 10^{4}$ dislocation lines per $\mathrm{cm}^{2}$ ).

A cellular calculation, when modified to fit the assumption of a random distribution of impurities, gives an order of magnitude estimate for the onset of concentration broadening at low temperatures. Broadening of a $2 \mathrm{p}$ state is predicted to start at about $3 \times 10^{15}$ impurities per $\mathrm{cm}^{3}$, if one replaces the mean spacing $\left(r_{s}\right)$ between impurities by an effective mean spacing of $0.7 \mathrm{r}_{s}$, and takes $a^{*}$ equal to $24 \AA$ in the expression $e^{-r / 2 a^{*}}$ occurring in the "2p" wave function under consideration. (This value of a* gives the experimental binding energy of $6.3 \times 10^{-3}$ ev for this state). Considering the crudeness of some of the approximations involved, in particular the use of hydrogenic wave functions, the agreement between theory and experiments seems to be rather good. An important question regarding the theoretical approach chosen in this problem, concerns the validity of the assumption of a random distribution of impurities. This assumption is believed to be more realistic than that of a regular array of impurities, and it is the only possible one in the absence of any concrete evidence for a clustering of boron impurities in silicon. 
$\underline{\text { Table } 1}$ : Standard Voigt Profiles

\begin{tabular}{|c|c|c|c|c|c|c|c|c|c|c|c|c|c|c|c|}
\hline \multicolumn{5}{|c|}{ Parameters } & \multicolumn{11}{|c|}{ Ordinates in Terms of Central Ordinate } \\
\hline \multirow[b]{2}{*}{$\beta_{1} / h$} & \multirow[b]{2}{*}{$\beta_{1} / \beta_{2}$} & \multirow{2}{*}{$\beta_{2} / h$} & \multirow{2}{*}{$\mathrm{B}_{2}^{2} / \mathrm{h}^{2}$} & \multirow[b]{2}{*}{ p } & 0.8 & 0.7 & 0.6 & 0.5 & 0.4 & 0.3 & 0.2 & 0.1 & 0.05 & 0.02 & 0.01 \\
\hline & & & & & \multicolumn{11}{|c|}{ Widths in Terms of Half-width $\left(b_{i} / h\right)$} \\
\hline 0.000 & 0.00 & 0.60 & 0.36 & 1.06 & 0.57 & 0.72 & 0.86 & 1.00 & 1.15 & 1.32 & 1.52 & 1.82 & 2.08 & 2.38 & 2.58 \\
\hline 0.025 & 0.04 & 0.59 & 0.34 & 1.08 & 0.56 & 0.72 & 0.86 & 1.00 & 1.15 & 1.33 & 1.53 & 1.84 & 2.12 & 2.49 & 2.82 \\
\hline 0.050 & 0.09 & 0.57 & 0.32 & 1.11 & 0.56 & 0.71 & 0.86 & 1.00 & 1.15 & 1.33 & 1.54 & 1.87 & 2.19 & 2.63 & 3.13 \\
\hline 0.075 & 0.14 & 0.55 & 0.31 & 1.13 & 0.56 & 0.71 & 0.86 & 1.00 & 1.16 & 1.33 & 1.56 & 1.90 & 2.25 & 2.79 & 3.56 \\
\hline 0.100 & 0.19 & 0.54 & 0.29 & 1.16 & 0.56 & 0.71 & 0.86 & 1.00 & 1.16 & 1.34 & 1.57 & 1.94 & 2.34 & 3.00 & 4.08 \\
\hline 0.125 & 0.24 & 0.52 & 0.27 & 1.18 & 0.56 & 0.71 & 0.86 & 1.00 & 1.17 & 1.34 & 1.59 & 1.98 & 2.42 & 3.24 & 4.58 \\
\hline 0.150 & 0.30 & 0.50 & 0.25 & 1.20 & 0.55 & 0.71 & 0.85 & 1.00 & 1.17 & 1.35 & 1.60 & 2.02 & 2.54 & 3.52 & 5.05 \\
\hline 0.175 & 0.36 & 0.48 & 0.23 & 1.23 & 0.55 & 0.70 & 0.85 & 1.00 & 1.17 & 1.36 & 1.62 & 2.06 & 2.64 & 3.80 & 5.50 \\
\hline 0.200 & 0.43 & 0.46 & 0.21 & 1.25 & 0.55 & 0.70 & 0.85 & 1.00 & 1.18 & 1.37 & 1.64 & 2.10 & 2.75 & 4.14 & 5.96 \\
\hline 0.225 & 0.51 & 0.44 & 0.20 & 1.28 & 0.54 & 0.70 & 0.85 & 1.00 & 1.18 & 1.38 & 1.66 & 2.15 & 2.87 & 4.44 & 6.40 \\
\hline & 0.59 & 0.42 & 0.18 & 1.30 & 0.54 & 0.70 & 0.84 & 1.00 & 1.18 & 1.39 & 1.68 & 2.19 & 2.98 & 4.73 & 6.78 \\
\hline & 0.69 & 0.40 & 0.16 & 1.33 & 0.53 & 0.69 & 0.84 & 1.00 & 1.19 & 1.40 & 1.71 & 2.24 & 3.12 & 5.03 & 7.15 \\
\hline 0.300 & 0.79 & 0.38 & 0.14 & 1.35 & 0.53 & 0.69 & 0.84 & 1.00 & 1.19 & 1.41 & 1.74 & 2.29 & 3.26 & 5.32 & 7.52 \\
\hline 0.325 & 0.92 & 0.35 & 0.12 & 1.38 & 0.53 & 0.68 & 0.84 & 1.00 & 1.19 & 1.42 & 1.77 & 2.34 & 3.39 & 5.57 & 7.86 \\
\hline 0.350 & 1.07 & 0.33 & 0.11 & 1.40 & 0.52 & 0.68 & 0.84 & 1.00 & 1.20 & 1.44 & 1.81 & 2.40 & 3.54 & 5.83 & 8.21 \\
\hline 0.375 & 1.26 & 0.30 & 0.09 & 1.43 & 0.52 & 0.68 & 0.83 & 1.00 & 1.20 & 1.45 & 1.85 & 2.46 & 3.70 & 6.07 & 8.55 \\
\hline 0.400 & 1.50 & 0.27 & 0.07 & 1.45 & 0.52 & 0.67 & 0.83 & 1.00 & 1.21 & 1.47 & 1.88 & 2.54 & 3.85 & 6.30 & 8.86 \\
\hline 0.425 & 1.83 & 0.23 & 0.05 & 1.48 & 0.51 & 0.67 & 0.83 & 1.00 & 1.21 & 1.48 & 1.92 & 2.64 & 4.00 & 6.55 & 9.18 \\
\hline 450 & 2.38 & 0.19 & 0.04 & 1.51 & 0.51 & 0.66 & 0.82 & 1.00 & 1.22 & 1.50 & 1.96 & 2.74 & 4.13 & 6.76 & 9.50 \\
\hline & 3. & 0.13 & 0.02 & 1.54 & 0.51 & 0.66 & 0.82 & 1.00 & 1.22 & 1.52 & 1.98 & 2.87 & 4.25 & 6.92 & 9.77 \\
\hline & & 0.00 & 0.00 & 1.57 & 0.50 & 0.66 & 0.82 & 1.00 & 1.22 & 1.53 & 2.00 & 3.00 & 4.36 & 7.00 & 9.95 \\
\hline
\end{tabular}


Table 2. Integrated absorption, $A\left(\mathrm{~cm}^{-2}\right)$

\begin{tabular}{cccccc}
\hline $\begin{array}{c}\text { Resistivity } \\
\text { (ohm cm) }\end{array}$ & \multicolumn{2}{c}{ Line No. } & \multicolumn{5}{c}{ Temperature $\left({ }^{\circ} \mathrm{K}\right)$} \\
(Fig. 1$)$ & 4.2 & 60 & 77 & 90 \\
\hline 130 & 1 & 1.4 & 1.8 & - & - \\
130 & 2 & 6.7 & 4.4 & - & - \\
130 & 4 & 6.6 & 5.3 & 1.8 & - \\
11 & 1 & 18 & 20 & 23 & - \\
11 & 2 & 54 & 109 & 50 & 53 \\
11 & 3 & 6.6 & - & - & - \\
11 & 4 & 44 & 73 & 60 & 45 \\
1.3 & 1 & 160 & 220 & 250 & 470 \\
1.3 & 2 & 630 & 920 & 600 & 650 \\
1.3 & 4 & 620 & 800 & 810 & 950 \\
\hline
\end{tabular}

Table 3. Integrated absorption cross-section, $\Sigma\left(10^{-14} \mathrm{~cm}\right)$.

\begin{tabular}{cccccc}
\hline $\begin{array}{c}\text { Resistivity } \\
\text { (ohm cm })\end{array}$ & Line No. & \multicolumn{5}{c}{ Temperature $\left({ }^{\circ} \mathrm{K}\right)$} \\
130 & 1 & 1.4 & 4.1 & - & - \\
130 & 2 & 6.7 & 10 & - & - \\
130 & 4 & 6.6 & 12 & 17 & - \\
11 & 1 & 1.5 & 2.2 & 4.3 & - \\
11 & 2 & 4.5 & 12 & 9.4 & 19 \\
11 & 3 & 0.55 & - & - & - \\
11 & 4 & 3.7 & 7.9 & 11 & 16 \\
1.3 & 1 & 1.3 & 2.0 & 2.9 & 7.2 \\
1.3 & 2 & 5.2 & 8.4 & 6.9 & 10 \\
1.3 & 4 & 5.2 & 7.3 & 9.3 & 15 \\
\hline
\end{tabular}


Appendix I: Distribution of holes between the ground state, excited states, and the valence band as a function of temperature.

The present appendix outlines the formalism used to calculate the curves shown in Figure 9. Let us begin by assuming that the number of donor impurities and free electrons is negligible. There are $\mathrm{N}_{\mathrm{A}}$ acceptor impurities with energy levels $E=-\epsilon_{a}$ above the valence band, and $p$ free holes. The probability $P(E)$ of an acceptor level having four electrons with paired spins when an extra electron is attached (ionized condition) is given by

$$
P(E)=\left\{1+2 \exp \cdot\left[\left(E-E_{F}\right) / k T\right]\right\}^{-1},
$$

where $E_{F}$ is the Fermi energy. The number of ionized acceptors is thus given by

$$
p=N_{A}\left\{1+2 \exp \cdot\left[\left(\epsilon_{a}+E_{F}\right) / k T\right]\right\}^{-1} \text {. }
$$

Writing

$$
p=N_{v} \exp \cdot\left(E_{F} / k T\right),
$$

one obtains a quadratic equation for $p$, whose appropriate solution is

$$
p=-N_{v}^{\prime} / 2+(1 / 2)\left(N_{v}^{\prime} 2+4 N_{v}^{\prime} N_{A}\right)^{1 / 2} \text {. }
$$

Here $N_{v}^{\prime}=\left(N_{v} / 2\right)$ exp. $\left(-\epsilon_{a} / k T\right)$, and the effective density of states $N_{v}$ is given by

$$
\mathrm{N}_{\mathrm{v}}=2\left(2 \pi \mathrm{kT} / \mathrm{h}^{2}\right)^{3 / 2}\left(\mathrm{~m}_{\mathrm{h} 1}{ }^{3 / 2}+\mathrm{m}_{\mathrm{h} 2}{ }^{3 / 2}\right) \text {. }
$$

Eq. (I-5) has been derived under the assumption that the actual energy surfaces in silicon may be approximated by two spherical constant-energy surfaces with effective masses $m_{h 1}$ and $m_{h 2}$. It was assumed that $\left(E_{F}-E\right) / k T>1$. This approximation is good as long as $p<0.4 N_{v}$ (Smith 1958), which applies for all cases of interest in this thesis. The valence band may be regarded to this approximation as a single level with 
degeneracy $\mathrm{N}_{\mathrm{V}}$, placed at the top of the band.

In deriving Eq. (I-2) from the expression for the probability of occupation: $P(E)$, it was assumed that one is only concerned with a single level. However, the impurity. levels have excited states, and these should be included in. Eq. (I-2) with the appropriate probabilities. One then has for the number of un-1onized acceptors

$$
N_{A-P}-p=N_{A} \cdot \sum_{i=0}^{m} 1+g_{i}^{-1} \exp \cdot\left[\left(E_{F}-E_{i}\right) / k T\right] \text {, }
$$

where $E_{i}$ is the energy of the $i$ th excited state, and $g_{i}$ is a number to take account of degeneracy and $\operatorname{spin}\left(E_{0}=-\epsilon_{a}, g_{0}=2\right)$. Since below $90^{\circ} \mathrm{K}$ one has $\left(E_{1}-E_{0}\right) / k T \gg 1$, the first term in the sumpredominates, and hence Eq. (I-2) is a good approximation.

Taking for the effective masses the values (Bube 1960)

$$
\mathrm{m}_{\mathrm{h} 1}=0.5 \mathrm{~m}_{\mathrm{e}} ; \mathrm{m}_{\mathrm{h} 2}=0.16 \mathrm{~m}_{\mathrm{e}} \text {, }
$$

Eq. (I-5) leads to

$$
\mathrm{N}_{\mathrm{v}}=2.02 \times 10^{15} \mathrm{~T}^{3 / 2} \mathrm{~cm}^{-3}
$$

Substitution into Eq. (I-4), taking the ionization energy of $\epsilon_{a}=46 \mathrm{mev}$ for boron-doped silicon, leads to the curves of Figure 9 for the number of ionized impurities as a function of the absolute temperature $T$.

The number of bound carriers in excited states $\mathrm{N}_{\mathrm{ex}}$ is then computed from

$$
N_{e x}=\left(N_{A}-p\right) \sum_{i} G_{i} \exp \cdot\left(-\epsilon_{i} / k T\right),
$$

where the assumption was made that $\mathrm{N}_{\mathrm{ex}} \ll\left(\mathrm{N}_{\mathrm{A}}-\mathrm{p}\right)$. The energy differences $\epsilon_{i}$ between the $i$ th excited state and the ground state are obtained from Figure 8. The degeneracies of the excited states relative to the ground state, $G_{1}$, were taken as $1,1,1 / 2,1 / 2$, for the lowest four excited states 
(Kohn 1957), which make the main contribution to $\mathrm{N}_{\mathrm{ex}}$. A value of $G_{1}$ equal to one was taken for the remaining five experimentally observed energy levels. (Figure 8 ).

The number of Impurities in the ground state, $\mathrm{N}_{g}$, was then obtained from

$$
\mathrm{N}_{\mathrm{g}}=\mathrm{N}_{\mathrm{A}}-\mathrm{p}-\mathrm{N}_{\mathrm{ex}}
$$


Appendix II: Second-order Stark shift for hydrogenic 1 s and $2 p$ states.

In the present appendix the matrix elements occurring in Chapter III, Eq. (6-2), will be evaluated:

$$
\left\langle F^{(1)},(\delta V)^{2} F^{(1)}\right\rangle \text {, }
$$

where $\delta V=-F e z$, and for $F^{(i)}$ hydrogenic wave functions $\psi_{n, 1, m}$, will be taken. In particular one has for the $1 \mathrm{~s}$ and the $2 \mathrm{p}$ states:

$$
\begin{aligned}
& \psi_{100}(r, \theta, \phi)=\pi^{-1 / 2} a^{*^{-3 / 2}} \exp \cdot\left(-r / a^{*}\right) \\
& \psi_{210}(r, \theta, \phi)=(1 / 4)(2 \pi)^{-1 / 2} a^{*}-5 / 2 r \cos \theta \exp \cdot\left(-r / 2 a^{*}\right) \\
& \psi_{21 \pm 1}(r, \theta, \phi)=(1 / 8) \pi^{-1 / 2} a^{*}-5 / 2 r \sin \theta \exp \cdot\left[\left(-r / 2 a^{*}\right) \pm 1 \phi\right] \cdot
\end{aligned}
$$

Hence one obtains

$$
\begin{aligned}
\left\langle\psi_{100},(\delta \mathrm{v})^{2} \psi_{100}\right\rangle & =F^{2} e^{2} \int_{0}^{2 \pi} d \phi \int_{0}^{\pi} d \theta \int_{0}^{\infty} d r r^{2} \sin \theta \psi_{100} r^{2} \cos ^{2} \theta \psi_{100}^{*} \\
& =2 F^{2} e^{2} a *^{-3} \int_{-1}^{1} w^{2} d w \int_{0}^{\infty} r^{4} \exp .(-2 r / a *) d r \\
& =a *^{2} .
\end{aligned}
$$

Similarly,

$$
\begin{aligned}
\left\langle\psi_{210},(\delta v)^{2} \psi_{210}\right\rangle & =(1 / 16) F^{2} e^{2} a^{-5} \int_{-1}^{1} w^{4} d w \int_{0}^{\infty} r^{6} \exp \cdot(-r / a *) d r \\
& =18 a *^{2} . \\
\left\langle\psi_{21 \pm 1},(\delta v)^{2} \psi_{21 \pm 1}\right\rangle & =(1 / 32) F^{2} e^{2} a *^{-5} \int_{-1}^{1} w^{2}\left(1-w^{2}\right) d w \int_{0}^{\infty} r^{6} \exp \cdot(-r / a *) d r \\
& =6 a *^{2} .
\end{aligned}
$$

Here $w=\cos \theta$. Thus one obtained Eq. (6-3) with $c$ given by 1,18 , and 6 for the 1 s state, $2 p_{0}$ state, and $2 p_{ \pm}$states respectively. 
Baltensperger, W. 1953. Ph1l. Mag. 44, 1355.

Bichard, J.W. and Giles, J.C. 1962. Can. J. Phys. 40, 1480.

Breene, Jr., R.G. 1957. Revs. Modern Phys. 29, 94.

Bube, R.H. 1960. Photoconductivity of Solids (John Wiley \& Sons, Inc.,

New York, London), p.209.

Burstein, E., Bell, E.E., Davisson, J.W., and Lax, M. 1953.

J. Phys. Chem. 57, 849 .

Burstein, E., Picus, G.S., Henvis, B., and Wallis, R. 1956.

J. Phys. Chem. Solids $\underline{1}, 65$.

Chandrasekhar, S. 1943. Revs. Modern Phys. 15, 1.

Colbow, K., Bichard, J.W., and Giles, J.C. 1962. Can. J. Phys. 40, 1436.

Colbow, K., 1962. Bull. Am. Phys. Soc., Series II, I, 485.

Conwel1, E.M. 1956. Phys. Rev. 103, 51.

Debye, P. and Hückel, E. 1923. Physik. Z. 24, 185.

Dexter, D.L., Zeiger, H.J., and Lax, B. 1956. Phys. Rev. 104, 637.

Dresselhaus, G., Kip, A.F., and Kittel, C. 1955. Phys. Rev. 98, 368.

Dresselhaus, G. 1955. Phys. Rev. 100, 580.

Ecker, G. 1957. Z. Physik 148, 593; 149, 254.

E11lott, R.J. 1954, Phys. Rev. 96, 266.

Herzberg, G. 1944. Atomic Spectra and Atomic Structure (Dover Publications,

New York), 2nd ed., p.51.

Holtsmark, J. 1919. Am. Physik, 58, 577; Physik. Z. 20, 162.

Holtsmark, J. 1924. Physik. 2. 25, 73.

Hrostowski, H.J., and Kaiser, R.H. 1958. J. Phys. Chem. Solids, 4, 148.

Irvin, I. C. 1962. Bell System Tech. Journ. 41, 387.

Kane, E.0. 1960. Phys. Rev. 119, 40.

Kittel, C. and Mitchell, A.H. 1954. Phys. Rev. 96, 1488. 
Kohn, W. and Luttinger, J.M. 1955. Phys. Rev. 97, 869.

Kohn, W. 1957. Solid State Physics, edited by F. Seltz and D. Turnbull, Vol. 5 (Acadmeic Press, Inc., New York), p. 257.

Lax, M. and Burstein, E. 1955. Phys.Rev. 100, 592.

London, F. and -Eisenschitz, R. 1930. Z. Physik 60, 491.

Margenau, H. 1933. Phys. Rev. 43, 129.

Margenau, H. and Murphy, G.M. 1943. The Mathematics of Physics and

Chemistry (Van Nostrand, New York), p. 559.

Margenau, H. and Lewis, M. 1959. Revs. Modern Phys. 31, 569.

Merck and Co., 1962. Private communication.

Morin, F.J. and Maita, J.P. 1954. Phys. Rev. 96, 28.

Moss, T.S. 1959. Optical properties of semiconductors (Butterworths, London), p.14.

Newman, R. 1956. Phys. Rev. 103, 103.

Nishikawa, K. 1962. Phys. Letters, 1, 140.

Nishikawa, K. and Barrie, R. 1962. Bull. Am. Phys. Soc., Series II, $7,485$.

Nishikawa, K. 1962. Ph.D. Thesis, University of British Columbia.

Plyler, E.K. and Acquista, N. 1956. J.Res. N.B.S., 56, 149.

Randa11, H.M., Dennison, D.M., Ginsburg, N. and .Weber, L.R. 1937.

Phys. Rev. 52, 160 .

Sampson, D. and Margenau, H. 1956. Phys. Rev. 103, 879.

Schechter, D. 1962. J. Phys. Chem. Solids, 23, 237.

Smith, R.A. 1958. Semiconductors (Cambridge University Press), p.80.

Unsö1d, A. 1955. Physik der Sternatmosphären (Springer-Verlag, Berlin, Göttingen,. Heidelberg), Chap. IX.

Van de Hulst, H.C. 1946. Bull. Astron. Inst. Neth. 10, 75 .

Van de Hulst, H.C. and Reesinck, I.I.M. 1947. Astrophys. J. 106, 121. Voigt, W. 1912. Münch. Ber. 603. 